\title{
Bulk-hinge correspondence and three-dimensional quantum anomalous Hall effect in second-order topological insulators
}

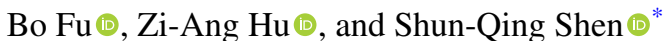 \\ Department of Physics, The University of Hong Kong, Pokfulam Road, Hong Kong, China
}

(Received 3 February 2021; revised 29 April 2021; accepted 7 July 2021; published 20 August 2021)

\begin{abstract}
The chiral hinge modes are the key feature of a second-order topological insulator in three dimensions. Here we propose a quadrupole index in combination of a slab Chern number in the bulk to characterize the flowing pattern of chiral hinge modes along the hinges at the intersection of the surfaces of a sample. We further utilize the topological field theory to present a picture of three-dimensional quantum anomalous Hall effect as a consequence of chiral hinge modes. The two bulk topological invariants can be measured in electric transport and magneto-optical experiments. In this way we establish the bulk-hinge correspondence in a three-dimensional second-order topological insulator.
\end{abstract}

DOI: 10.1103/PhysRevResearch.3.033177

\section{INTRODUCTION}

The bulk-boundary correspondence lies at the heart of topological states of matter and topological materials [1-4]. It bridges the topology of bulk band structures and the physical observables near the boundary. In the quantum Hall effect and quantum anomalous Hall effect (QAHE), the quantized Hall conductance is associated with the TKNN number of the band structure and the number of the edge modes of electrons around the boundary [5-8]. In a topological insulator, a $Z_{2}$ index in the bulk is associated with the number of the gapless Dirac cones of the surface electrons [9-11]. This reflects intrinsic attributes of the topological phenomena. A recent advance in the field of topological materials is the discovery of higher-order topological insulators [12-20]. A second-order topological insulator in three dimensions refers to an insulator with one-dimensional the chiral hinge modes (CHMs) localized on the hinges at the intersection of adjacent side surfaces [15-27]. Over the past few years, a great of efforts have been made to explore the possible relation of the bulk bands and existence of hinge modes as an extension of the bulk-boundary correspondence, such as effective mass analysis [18-23], the symmetry indicator [28-36], and spectral flow analysis [37]. All the approaches have their own merits. However, CHMs in a second-order topological insulator may display various flowing patterns as illustrated in Fig. 1. It lacks a systematic method to provide a comprehensive description of diverse flowing patterns. Also it is desirable to learn which observable in the bulk is associated with the CHMs.

\footnotetext{
*sshen@hku.hk

Published by the American Physical Society under the terms of the Creative Commons Attribution 4.0 International license. Further distribution of this work must maintain attribution to the author(s) and the published article's title, journal citation, and DOI.
}

In the present work, we address the bulk-hinge correspondence and three-dimensional (3D) QAHE as a physical consequence of the CHMs in a second-order topological insulator. We start with a minimal four-band model to reveal different flowing patterns of CHMs. It is found that a quadrupole index is associated with the flowing direction of four hinge modes of the system along one direction and a slab quantized Hall conductance reveals the formation of a closed loop of the CHMs. We further demonstrate the correspondent connection of the CHMs to the quadrupole index and the slab Chern number by means of topological field theory. Finally we propose to utilize magneto-optical Faraday and Kerr effects to detect these topological invariants.

\section{MODEL HAMILTONIAN AND SYMMETRY ANALYSIS}

We start with a minimal four-band Hamiltonian,

$$
\mathcal{H}=\mathcal{H}_{0}+\sum_{i=1}^{3} \mathcal{V}_{i}
$$

which consists of four parts (Appendix A). The primary part is

$$
\begin{aligned}
\mathcal{H}_{0}= & \hbar \sigma_{x}\left[v_{\perp}\left(k_{x} s_{x}+k_{y} s_{y}\right)+v_{z} k_{z} s_{z}\right] \\
& +\left[m_{0}+m_{\perp}\left(k_{x}^{2}+k_{y}^{2}\right)+m_{z} k_{z}^{2}\right] \sigma_{z} s_{0},
\end{aligned}
$$

where $k_{x}, k_{y}, k_{z}$ are the wave vectors, and $m_{i}$ and $v_{i}$ are the model parameters. We consider the basis functions $(|+\uparrow\rangle$, $|+\downarrow\rangle,|-\uparrow\rangle,|-\downarrow\rangle)$ with \pm being the parity eigenvalues and $\uparrow(\downarrow)$ denoting the spin-up (-down) state, thus $\boldsymbol{s}_{i}$ and $\sigma_{i}$ $(i=x, y, z)$ are the Pauli matrices and $s_{0}$ and $\sigma_{0}$ the identity matrices acting in spin and orbital space, respectively. The point group of $\mathcal{H}_{0}$ is $D_{4 h}$, which can be generated by a fourfold rotation $\mathcal{R}_{4}^{z}$ around $z$ axis, a twofold rotation $\mathcal{R}_{2}^{x}$ around $x$ axis and the spatial inversion $\mathcal{I}$. $\mathcal{H}_{0}$ possesses the time reversal symmetry $\mathcal{T}=i s_{y} \mathcal{K}\left(\mathcal{T}^{2}=-1\right.$, and $\mathcal{K}$ is complex conjugate) and belongs to the symplectic symmetry class AII. Here we focus on the case of both $m_{0} m_{\perp}<0$ and $m_{0} m_{z}<0$ such that 
(a)

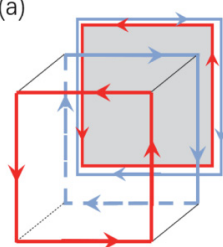

(b)

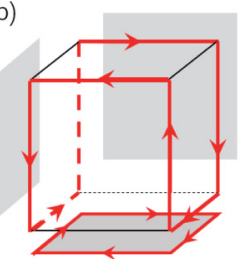

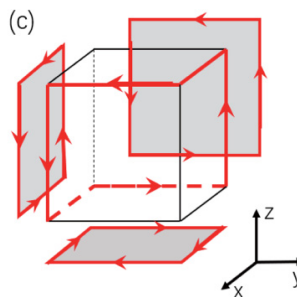

FIG. 1. Illustration of selected patterns of chiral hinge modes and their projection in a second-order topological insulator in three dimensions. (a) 3D antiferromagnetic quantum anomalous Hall insulator: A double-loop pattern with the quadrupole indices $\Delta_{x y}=$ $-\Delta_{z x}=1$ and $\Delta_{y z}=0$ and the slab Chern number $n_{x}=n_{y}=n_{z}=$ 0. (b) 3D rotoinversion quantum anomalous Hall insulator: A singleloop pattern with $\Delta_{x y}=1$ and $\Delta_{y z}=\Delta_{z x}=0$ and $n_{x}=n_{y}=0$ and $n_{z}=-1$. (c) $3 \mathrm{D}$ inversion quantum anomalous Hall insulator: A single-loop pattern with $\Delta_{x y}=\Delta_{y z}=\Delta_{z x}=0$ and $n_{x}=n_{y}=$ $-n_{z}=1$.

$\mathcal{H}_{0}$ describes a 3D strong topological insulator with gapless Dirac cone of the surface states at all surfaces [4,38]. $\mathcal{H}_{0}$ also respects the global chiral symmetry $\mathcal{C}=\sigma_{y} s_{0}$, i.e., $\left\{\mathcal{C}, \mathcal{H}_{0}\right\}=$ 0 . Including the crystalline symmetries, the total point symmetry group is $\mathcal{G}_{0}=D_{4 h} \times\{1, \mathcal{T}, \mathcal{P}, \mathcal{C}\}$ with the particle-hole symmetry $\mathcal{P} \equiv \mathcal{C} \mathcal{T}^{-1}$ [39]. As shown below all the terms in $\mathcal{H}$ preserve $\mathcal{P}$, it is more convenient to rewrite $\mathcal{G}_{0}$ as $\mathcal{G}_{0}=\widetilde{\mathcal{G}}_{0} \times$ $\{1, \mathcal{P}\}$ with the magnetic group $\widetilde{\mathcal{G}}_{0}=D_{4 h} \times\{1, \mathcal{T}\}=D_{4 h} \oplus$ $\mathcal{T} D_{4 h}\left(\right.$ or $\left.4 / m m 1^{\prime}\right)$. The three additional terms breaks different symmetries, respectively. $\mathcal{V}_{1}=c\left(k_{x}^{2}-k_{y}^{2}\right) \sigma_{y} s_{0}$ breaks both $\mathcal{T}$ and $\mathcal{R}_{4}^{z}$ individually, but respects their combination $\mathcal{R}_{4}^{z} \mathcal{T}$. $\mathcal{V}_{2}=d \sigma_{y} s_{0}$ is an antiferromagnetic term which breaks both $\mathcal{T}$ and $\mathcal{I}$, but respects the combination $\mathcal{I} \mathcal{T} . \mathcal{V}_{3}=\sum_{i=x, y, z} b_{i} s_{i}$ is the magnetic Zeeman interaction or ferromagnetic term which breaks $\mathcal{T}$ but preserves $\mathcal{I}$. The group $\widetilde{\mathcal{G}_{0}}$ is a HeeschSchubnikov magnetic group of type II; the inclusion of any the term in $\mathcal{V}_{i}$ breaks time reversal symmetry and several spatial symmetries and reduces it to a magnetic group of type III. In general, the reduced magnetic point group can be expressed as [39] $\widetilde{\mathcal{G}}_{i}=N \oplus \mathcal{T}(G-N)$, where $G$ is subgroup of $D_{4 h}$ and $N$ is a halving subgroup of $G$. Combination of the three terms $\mathcal{V}_{i}$ may generate higher-order topological phases with different symmetries (Appendix B).

(i) 3D chiral higher-order topological insulator (CHOTI): $\mathcal{H}_{\text {CHOTI }}=\mathcal{H}_{0}+\mathcal{V}_{1}$. The presence of $\mathcal{V}_{1}$ reduces the magnetic group to $\widetilde{\mathcal{G}}^{\prime}=D_{2 d} \oplus \mathcal{T}\left(D_{4 h}-D_{2 d}\right)$. The term opens an gap with opposite sign for the surface states on the neighboring surfaces parallel to $z$ axis and the CHMs may be localized at their intersections. The CHMs are protected by the combination of fourfold rotational symmetry and time-reversal symmetry $\mathcal{R}_{4}^{z} \mathcal{T}$. The surface states on the bottom (00) $\left.\overline{1}\right)$ and top (001) surface remain gapless.

(ii) 3D antiferromagnetic quantum anomalous Hall insulator (QAHI): $\mathcal{H}_{\mathrm{AQAHI}}=\mathcal{H}_{0}+\mathcal{V}_{1}+\mathcal{V}_{2}$. The inclusion of $\mathcal{V}_{2}$ in the Hamiltonian of CHOTI further reduces the magnetic group to $\widetilde{\mathcal{G}}_{1}=D_{2} \oplus \mathcal{T}\left(D_{2 h}-D_{2}\right)$. The $\mathcal{V}_{2}$ term anticommutes with the linear terms $\hbar v_{\perp}\left(k_{x} \sigma_{x} s_{x}+k_{y} \sigma_{x} s_{y}\right)$ along $x$ and $y$ directions. Thus, it acts as the mass terms and gap out the surface states on (001) and (001) while being projected onto the $x-y$ surface. Since $\mathcal{V}_{2}$ commutes with the mass term $\sigma_{y} s_{0}$ for the

surface states parallel with the $z$ axis induced by $\mathcal{V}_{1}$, it only modifies its value and has no influence on the four hinge states along the $z$ direction. When all the surface states are gapped out and the Fermi level is located in the surface band gap, the electrons can only propagate unidirectionally along the hinges shared by adjacent side surfaces due to time reversal symmetry breaking. The presence of the antiunitary symmetry $\mathcal{I} \mathcal{T}$ imposes the strong restriction that there must be another hinge state propagating in the same direction on its spatial inversion if one CHMs propagates along any hinge. Thus, the CHMs may form two closed loops on the surfaces (100) and (100) as shown in Fig. 1(a). The relative sign between $c$ in $\mathcal{V}_{1}$ and $d$ in $\mathcal{V}_{2}$ will determine which surface the two hinge mode loops locate around.

(iii) $3 \mathrm{D}$ rotoinversion QAHI: $\mathcal{H}_{\mathrm{RQAHI}}=\mathcal{H}_{0}+\mathcal{V}_{1}+\mathcal{V}_{3}$ with $\mathcal{V}_{3}=b_{z} s_{z}$. The inclusion of $\mathcal{V}_{3}$ with magnetic field in $z$ direction reduces the magnetic group to $\widetilde{\mathcal{G}}_{2}=S_{4} \oplus \mathcal{T}\left(D_{2 d}-\right.$ $\left.S_{4}\right)$. The ferromagnetic term $b_{z} s_{z}$ behaves similar to the antiferromagnetic term $\mathcal{V}_{2}$ but open gaps with different signs for the surface Dirac electrons of the 3D CHOTI on the (001) and $(00 \overline{1})$ surfaces. As a consequence, the chiral hinge modes of 3D rotoinversion QAHI will exhibit a distinctly different pattern compared with 3D antiferromagnetic QAHI. The presence of the improper rotation symmetry $\mathcal{S}_{4}=\mathcal{I R}_{4}^{z}$ protects a single-loop CHMs wriggling around the bulk as shown in Fig. 1(b). The relative sign between $b$ and $c$ determines the wriggling way of the single-loop CHMs.

(iv) 3D inversion QAHI: $\mathcal{H}_{\mathrm{IQAHI}}=\mathcal{H}_{0}+\mathcal{V}_{3}$ with $\mathcal{V}_{3}=$ $b \sum_{i=x, y, z} s_{i}$. Only in the presence of $\mathcal{V}_{3}$ that magnetic field points to (111) direction, i.e., $b_{x}=b_{y}=b_{z}=b$, the magnetic point group is $\widetilde{\mathcal{G}_{3}}=C_{i} \oplus \mathcal{T}\left(C_{2 h}-C_{i}\right)$. Due to the presence of the inversion symmetry $\mathcal{I}$, the CHMs at the inversion symmetric hinges are propagating in the opposite directions, and form a closed loop as shown in Fig. 1(c).

Here, we only take several higher-order topological phases with distinct flowing patterns of the hinge currents as examples to illustrate the bulk-hinge correspondence. All the possible patterns of hinge currents can be constructed by the strategy in Appendix $\mathrm{C}$ and the relationship between different patterns and the topological invariants are also checked.

\section{THE QUADRUPOLE INDEX}

To characterize the topological hinge modes, we introduce a quadrupole index as topological invariant. There are the CHMs along four hinges in the $z$ direction in the case of Figs. 1(a) and 1(b). The energy dispersions of the four hinge modes connect the conduction and valence bands, and cross at $k_{z}=0$ (see Fig. 4 in Appendix A). For a specific $k_{z}, \mathcal{H}\left(k_{z}\right)$ can be viewed as a 2D system in the $x-y$ plane and there are four corner states. The existence of corner states can be characterized by the quadrupole moment [40-43],

$$
q_{x y}\left(k_{z}\right)=\frac{1}{2 \pi} \operatorname{Im} \log \left[\operatorname{det}\left[U_{k_{z}}^{\dagger} Q_{x y} U_{k_{z}}\right] \sqrt{\operatorname{det} Q_{x y}^{\dagger}}\right],
$$

where the matrix $U_{k_{z}}$ is constructed by the occupied lowest energy states, $Q_{x y}=e^{2 \pi i \hat{r}_{x} \hat{r}_{y} / L_{x} L_{y}}, \hat{r}_{\alpha}$ are the position operators, and $L_{\alpha}$ are the lengths of the system in the $\alpha$ direction. For the occupied states, we have $\mathcal{H}\left(k_{z}\right) U_{k_{z}}=U_{k_{z}} E_{\text {occ }}\left(k_{z}\right)$, with $E_{\text {occ }}\left(k_{z}\right)=\operatorname{diag}\left[\epsilon_{1}\left(k_{z}\right), \epsilon_{2}\left(k_{z}\right), \ldots \epsilon_{N_{\text {occ }}}\left(k_{z}\right)\right] . \epsilon_{n}$ and $\left|u_{k_{z}}^{n}\right\rangle$ 
are the energy eigenvalue and eigenstate, $\mathcal{H}\left(k_{z}\right)\left|u_{k_{z}}^{n}\right\rangle=$ $\varepsilon_{n}\left(k_{z}\right)\left|u_{k_{z}}^{n}\right\rangle . U_{k_{z}}$ is constructed by the occupied states $U_{k_{z}}=$ $\left(\left|u_{k_{z}}^{1}\right\rangle, \ldots\left|u_{k_{z}}^{N_{\text {occ }}}\right\rangle\right)$, which has dimension of $N_{\text {tot }} \times N_{\text {occ }} \cdot N_{\text {tot }}=$ $L_{x} \times L_{y} \times N_{\text {orb }}$ denotes the dimension of total Hilbert space with $N_{\text {orb }}$ being the "orbitals" per site and $N_{\text {occ }}$ denotes the number of occupied bands. We hence build the the projection operator into the occupied energy bands $P_{\text {occ }}=U U^{\dagger}$ and we also have relation $U^{\dagger} U=1_{N_{\text {occ }} \times N_{\text {occ }}}$. The determinant can be reformed as

$$
\operatorname{det}\left[U_{k_{z}}^{\dagger} Q U_{k_{z}}\right]=\operatorname{det}\left[1_{N_{\mathrm{occ}} \times N_{\mathrm{occ}}}+U_{k_{z}}^{\dagger}(Q-1) U_{k_{z}}\right]
$$

Since the Hilbert space of the Hamiltonian (occupied and unoccupied energy bands included) at each $k_{z}$ is complete, we have the relation $V_{k_{z}} V_{k_{z}}^{\dagger}+U_{k_{z}} U_{k_{z}}^{\dagger}=1$ where we denote $V_{k_{z}}$ as the eigenstates for unoccupied band. By using the Sylvester's determinant identity, we have

$$
\operatorname{det}\left[U_{k_{z}}^{\dagger} Q U_{k_{z}}\right]=\operatorname{det}\left[V_{k_{z}}^{\dagger} Q^{\dagger} V_{k_{z}}\right] \operatorname{det}[Q] \text {. }
$$

We then focus on the anti-symmetries (e.g., the chiral symmetry $\mathcal{C}$ or particle-hole symmetry $\mathcal{P}$ ) because these symmetries will relate the occupied states to the unoccupied states which is essential in the quantization of the quadrupole moment [42]. Under the antisymmetry $\mathcal{O}_{a}$, the Hamiltonian obeys

$$
\mathcal{O}_{a} \mathcal{H}\left(k_{z}\right) \mathcal{O}_{a}^{-1}=-\mathcal{H}\left(\chi k_{z}\right),
$$

with $\chi=-1$ denotes the operation $\mathcal{O}_{a}$ will inverse the momentum $k_{z}$ and $\chi=+1$ means that $\mathcal{O}_{a}$ keeps $k_{z}$ unchanged. Following the same procedure in Ref. [42], it is found that an antisymmetry $\mathcal{O}_{a}$ leaves $x y$ plane invariant $\mathcal{O}_{a} \mathcal{H}\left(k_{z}\right) \mathcal{O}_{a}^{-1}=$ $-\mathcal{H}\left(-k_{z}\right)$ which will impose a constraint on the quadrupole moment $q_{x y}\left(k_{z}\right): q_{x y}\left(k_{z}\right)+q_{x y}\left(-k_{z}\right)=0$ or 1 . At two high symmetry points $\Lambda_{z}=0$ or $\pi$, the symmetry is restored, $\mathcal{O}_{a} \mathcal{H}\left(\Lambda_{z}\right) \mathcal{O}_{a}^{-1}=-\mathcal{H}\left(\Lambda_{z}\right)$, and $q_{x y}\left(\Lambda_{z}\right)$ must be quantized to 0 or $\frac{1}{2}$. Nonzero quantized $q_{x y}\left(\Lambda_{z}\right)$ indicates the system topologically nontrivial and the existence of four zero-energy corner states in the reduced 2D subspace. For example, if $q_{x y}\left(k_{z}=0\right)=1 / 2$, then $q_{x y}( \pm \pi)=0$ or 1 . In this case, there exist CHMs which compensate for the difference of the corner charges. Thus, we can introduce a quadrupole index,

$$
\Delta_{x y}=\int_{0}^{2 \pi} d k_{z} \partial_{k_{z}} q_{x y}\left(k_{z}\right),
$$

to characterize the existence and the flowing direction of four CHMs. For antiferromagnetic QAHI in Fig. 1(a), we have $\Delta_{x y}=-\Delta_{z x}=1$ and $\Delta_{y z}=0$, which are protected by the combination of chiral symmetry and the mirror symmetry $\mathcal{C M}_{\alpha}$ and the combination of chiral symmetry and the time reversal symmetry $\mathcal{C} \mathcal{T}$. For rotoinversion QAHI in Fig. 1(b), we have $\Delta_{x y}=1$ and $\Delta_{y z}=\Delta_{z x}=0$. The quadrupole index along the $z$ direction is protected only by $\mathcal{C} \mathcal{T}$ and along the $x$ (y) is protected by both $\mathcal{C} \mathcal{M}_{x(y)}$ and $\mathcal{C} \mathcal{T}$. For inversion QAHI in Fig. 1(c), $\Delta_{x y}=\Delta_{y z}=\Delta_{z x}=0$.

\section{THE SLAB CHERN NUMBER}

The slab Chern number is another topological invariant as the quadrupole index alone are not enough to characterize the diversity of the flowing pattern of the CHMs. Consider a slab geometry of the sample with a finite thickness $L_{z}$ with the periodic boundary condition along the $\mathrm{x}$ and $\mathrm{y}$ direction. Denote the Bloch eigenstates by $\left|u_{n}\left(\boldsymbol{k}_{\perp}, z\right)\right\rangle$ are the Bloch eigenstates, $\mathcal{H}\left(\boldsymbol{k}_{\perp}, z\right)\left|u_{n}\left(\boldsymbol{k}_{\perp}, z\right)\right\rangle=\varepsilon_{n}\left(\boldsymbol{k}_{\perp}\right)\left|u_{n}\left(\boldsymbol{k}_{\perp}, z\right)\right\rangle$ with $\boldsymbol{k}_{\perp}=\left(k_{x}, k_{y}\right)$ and the index $n$ for the bands. The space-resolved Berry connection is given by $\mathcal{A}_{\alpha ; n, n^{\prime}}\left(\boldsymbol{k}_{\perp}, z\right)=$ $-i\left\langle u_{n}\left(\boldsymbol{k}_{\perp}, z\right)\left|\partial_{\alpha}\right| u_{n^{\prime}}\left(\boldsymbol{k}_{\perp}, z\right)\right\rangle$ for the two occupied bands $n, n^{\prime}$. In this way we define the slab Hall conductance and its relation to a slab Chern number $n_{z}$ [36],

$$
\sigma_{x y}^{\text {slab }}=\int_{0}^{L_{z}} d z \sigma_{x y}(z)=n_{z} \frac{e^{2}}{h},
$$

where $\sigma_{x y}(z)=\frac{e^{2}}{2 \pi h} \int d^{2} \boldsymbol{k}_{\perp} \operatorname{Tr}\left[\mathcal{F}_{x y}\left(\boldsymbol{k}_{\perp}, z\right)\right]$ and

$$
\mathcal{F}_{\alpha \beta}(\boldsymbol{k})=\partial_{\alpha} \mathcal{A}_{\beta}(\boldsymbol{k})-\partial_{\beta} \mathcal{A}_{\alpha}(\boldsymbol{k})+i\left[\mathcal{A}_{\alpha}(\boldsymbol{k}), \mathcal{A}_{\beta}(\boldsymbol{k})\right]
$$

is the non-Abelian Berry curvature in terms of $\mathcal{A}_{\alpha ; n, n^{\prime}}\left(\boldsymbol{k}_{\perp}, z\right)$. Because of the periodicity of the Berry connection in the first Brillouin zone, it can be proved that the slab Chern number $n_{z}$ is quantized if the filled bands has a band gap to the excited states for a band insulator. According to the bulk-boundary correspondence [8], each nonzero Chern number is associated with the closed loop of chiral edge state. In Fig. 1(a), for antiferromagnetic QAHI we have $n_{x}=n_{y}=n_{z}=0$, while two quadrupole indices are not vanishing $\Delta_{x y}=-\Delta_{z x}=1$. The system in a slab geometry (the open boundary condition is imposed in the $y$ direction) is analog to the quantum spin Hall insulator except the the two counter-propagating hinges modes are localized on the opposite sides. Experimentally, the quantized anomalous Hall effect can be measured by using the surface-sensitive method [44]. In Fig. 1(b), for rotoinversion QAHI we have $n_{z}=-1$ and $n_{x}=n_{y}=0$. There is a closed loop of chiral edge mode around the $z$ axis. Combined with the nonzero quadrupole index $\Delta_{x y}=1$. there are four CHMs along the four hinges along the $z$ axis, the two indices can determine that a single-loop of CHMs that wriggles around the bulk. QAHE can be detected through a global quantum Hall measurement probing the whole sample due to the nonzero $n_{z}$. In Fig. 1(c), for inversion QAHI we have $n_{x}=n_{y}=-n_{z}=1$. There is a single loop of chiral edge mode around each axis. Because of the zero quadrupole indices around the three axes, there is no four CHMs along one direction. It exhibits a single CHM traversing half of its hinges, which can be projected out a single closed loop in the direction of $x, y$, and $z$. The QAHE can be observed for three directions due to the nonvanishing slab Chern numbers.

\section{3D QUANTUM ANOMALOUS HALL EFFECT}

The CHMs can be further understood in the framework of topological field theory with an effective action [45],

$$
\mathcal{S}=\int d^{3} r d t\left[\frac{1}{8 \pi}\left(\epsilon \mathbf{E}^{2}-\frac{1}{\mu} \mathbf{B}^{2}\right)+\frac{\theta(\mathbf{r}, t) e^{2}}{4 \pi^{2} \hbar c} \mathbf{E} \cdot \mathbf{B}\right],
$$

where $\mathbf{E}$ and $\mathbf{B}$ are the electromagnetic fields, and $\epsilon$ and $\mu$ are the dielectric constant and magnetic permeability, respectively. $\theta(\mathbf{r}, t)$ is known as the axion angle [46]. The product $\mathbf{E} \cdot \mathbf{B}$ is odd under the time reversal or spatial inversion, $\theta$ has to be 0 (modulo $2 \pi$ ) for a trivial insulator and the vacuum and $\pi$ for a topological insulator with respect to the symmetries $[47,48]$. In the quadratic order of electric and magnetic fields, 
besides the Maxwell term, the $\theta$ term may give rise to the topologically magnetoelectric effect that an electric field can induce a magnetic field and vice verse [45,49-53]. By taking the functional derivative of $\theta$ term with respect to a gauge field, the induced electric current density depends on the spatial and temporal gradients of the $\theta$-field $[45,46]$,

$$
\mathbf{j}_{\theta}(\mathbf{r}, t)=\frac{e^{2}}{2 \pi h}\left[\partial_{t} \theta(\mathbf{r}, t) \mathbf{B}-\nabla \theta(\mathbf{r}, t) \times \mathbf{E}\right] .
$$

The first term depends on the temporal gradient of the $\theta$-field and is proportional to magnetic field, i.e., the so-called chiral magnetic field, and vanishes in a static limit. The second term depends spatial gradient of the $\theta$ field and is perpendicular to the electric field, i.e., the anomalous Hall effect. Thus, there will be surface anomalous Hall effect at the interface between two regions with different $\theta$ values and no Hall response will exist in the bulk as $\theta$ takes a constant value $\theta_{b}[54,55]$.

The value of $\theta_{b}$ is given by the three-dimensional integration of the Chern-Simons 3-form over momentum space [56,57],

$$
\frac{\theta_{b}}{2 \pi}=\int \frac{d^{3} k}{16 \pi^{2}} \epsilon_{\alpha \beta \gamma} \operatorname{tr}\left[\mathcal{F}_{\alpha \beta}(\boldsymbol{k}) \mathcal{A}_{\gamma}(\boldsymbol{k})-\frac{i}{3}\left[\mathcal{A}_{\alpha}(\boldsymbol{k}), \mathcal{A}_{\beta}(\boldsymbol{k})\right] \mathcal{A}_{\gamma}(\boldsymbol{k})\right] .
$$

Consider a Bloch Hamiltonian $\mathcal{H}(\boldsymbol{k})$ which is invariant under $\mathcal{O}$, the eigenstates of $\mathcal{H}(\boldsymbol{k})$ at $\boldsymbol{k}$ and $D_{\mathcal{O}} \boldsymbol{k}$ must be related by a gauge transformation, where $D_{\mathcal{O}}$ is an operator transforming $\boldsymbol{k}$ to $D_{\mathcal{O}} \boldsymbol{k}$ in momentum space with the Jacobian matrix as $J_{a b}=$ $\partial\left(D_{\mathcal{O}} k\right)_{b} / \partial k_{a}$. We can prove that only those symmetries with Jacobian determinant det $J=-1$ will quantize the axion angle. There are two types symmetries satisfying this condition: the improper rotational symmetries and the combined symmetries from time reversal symmetry and the proper rotation symmetry, which will put the following constraints on axion angle $\theta_{b}$, respectively,

$$
2 \frac{\theta_{b}}{2 \pi}=\int \frac{d^{3} k}{24 \pi^{2}} \epsilon_{\alpha \beta \gamma} \operatorname{tr}\left[\left(U^{*}(\boldsymbol{k}) \partial_{\alpha} U^{T}(\boldsymbol{k})\right)\left(U^{*}(\boldsymbol{k}) \partial_{\beta} U^{T}(\boldsymbol{k})\right)\left(U^{*}(\boldsymbol{k}) \partial_{\gamma} U^{T}(\boldsymbol{k})\right)\right],
$$

and

$$
2 \frac{\theta_{b}}{2 \pi}=\int \frac{d^{3} k}{24 \pi^{2}} \epsilon_{\alpha \beta \gamma} \operatorname{tr}\left[\left(U(\boldsymbol{k}) \partial_{\alpha} U^{\dagger}(\boldsymbol{k})\right)\left(U(\boldsymbol{k}) \partial_{\beta} U^{\dagger}(\boldsymbol{k})\right)\left(U(\boldsymbol{k}) \partial_{\gamma} U^{\dagger}(\boldsymbol{k})\right)\right] .
$$

* and $T$ represents the complex conjugate and transpose, respectively, and $U(\boldsymbol{k})$ is the unitary transformation matrix acting on the space of occupied bands, $\mathcal{O}\left|u_{n}(\boldsymbol{k})\right\rangle=\sum_{m} U_{n m}(\boldsymbol{k})\left|u_{m}\left(D_{\mathcal{O}} \boldsymbol{k}\right)\right\rangle$ with $n$ as the occupied band index. $U(\boldsymbol{k})$ is periodic in $k_{x}, k_{y}$, and $k_{z}$, and thus defines a map from a 3-torus to the space of $N_{\text {occ }} \times N_{\text {occ }}$ unitary matrices. Such maps are classified by an integer topological invariant or winding number which is given by the righthand side of the above equations, $2 \frac{\theta_{b}}{2 \pi} \in \mathbb{Z}$. If $U(\boldsymbol{k}) \in U(1)$, then the terms in the brackets can be interchanged, considering the antisymmetric form of the winding number, the axion angle $\theta_{b}$ always trivially vanishes.

Therefore to yield a nontrivial axion angle, the occupied bands need to be degenerate such that the gauge transformation $U(\boldsymbol{k})$ becomes non-Abelian. (i) For 3D antiferromagnetic QAHI $(\boldsymbol{b}=0, c \neq 0$, and $d \neq 0$ ), the all five $4 \times 4$ matrices in $\mathcal{H}$ anticommute. The eigenvalues for two valance bands are easily obtained as

$$
\varepsilon_{ \pm}^{v}(\boldsymbol{k})=-\sqrt{\boldsymbol{p}(\boldsymbol{k})^{2}+M(\boldsymbol{k})^{2}+\left[d+c\left(k_{x}^{2}-k_{y}^{2}\right)\right]^{2}},
$$

where $M(\boldsymbol{k})=m_{0}+m_{\perp}\left(k_{x}^{2}+k_{y}^{2}\right)+m_{z} k_{z}^{2}$ and $\boldsymbol{p}(\boldsymbol{k})=\left(v_{\perp} k_{x}, v_{\perp} k_{y}, v_{z} k_{z}\right)$. The two bands are doubly degenerate for the whole Brillouin zone which is guaranteed by the combined symmetry $\mathcal{T} \mathcal{I}$ (with $(\mathcal{I} \mathcal{T})^{2}=-1$ ). (ii) For the3D rotoinversion QAHI $\left(\boldsymbol{b}=\left(0,0, b_{z}\right), c \neq 0\right.$ and $\left.d=0\right)$, the eigenvalues for two valance bands are

$$
\varepsilon_{ \pm}^{v}(\boldsymbol{k})=-\sqrt{b_{z}^{2}+\boldsymbol{p}(\boldsymbol{k})^{2}+M(\boldsymbol{k})^{2} \pm 2 \sqrt{b_{z}^{2}\left[v_{z}^{2} k_{z}^{2}+M(\boldsymbol{k})^{2}+c^{2}\left(k_{x}^{2}-k_{y}^{2}\right)^{2}\right]}}
$$

The band degeneracy occurs only for topological nontrivial case and at the four momenta $\left( \pm \sqrt{-\frac{m_{0}}{2 m_{\perp}}}, \pm \sqrt{-\frac{m_{0}}{2 m_{\perp}}}\right.$ ). (iii) For the 3D inversion QAHI $\left(\boldsymbol{b}=\left(b_{x}, b_{y}, b_{z}\right)\right.$ and $\left.c=d=0\right)$, the eigenvalues for two valance bands are explicitly written as

$$
\varepsilon_{ \pm}^{v}(\boldsymbol{k})=-\sqrt{\boldsymbol{b}^{2}+\boldsymbol{p}(\boldsymbol{k})^{2}+M(\boldsymbol{k})^{2} \pm 2 \sqrt{[\boldsymbol{b} \cdot \boldsymbol{p}(\boldsymbol{k})]^{2}+\boldsymbol{b}^{2} M(\boldsymbol{k})^{2}}}
$$

The band degeneracy occurs at the momenta which satisfy both the conditions $M(\boldsymbol{k})=0$ and $\boldsymbol{b} \cdot \boldsymbol{p}(\boldsymbol{k})=0$. The first condition requires band topology is nontrivial $\left(m_{0} m_{\perp}, m_{0} m_{z}<\right.$ 0 ), and restricts the momenta to a $2 \mathrm{D}$ ellipsoidal momentum surface. The second condition further restrict the momenta to the line nodes perpendicular to the vector $\boldsymbol{b}$. For the case (i), the axion angle is not quantized and can take any value. For the cases (ii) and (iii), the axion angle are quan- tized and nontrivial. While for topological trivial insulators $\left(m_{0} m_{\perp}, m_{0} m_{z}>0\right)$, the band degeneracy is removed for all the Brillouin zone the axion angle must trivially vanish in this situation. We want to emphasize the band degeneracy for these two cases is not due to the symmetry but the band topology. Therefore, the band degeneracy is necessary but not sufficient for the quantized nontrivial axion angle. 
In addition to the inversion or time-reversal symmetry, $\theta_{b}$ will be quantized with improper rotation symmetries or a combination of time-reversal symmetry and proper rotation symmetries [58]. From Eq. (11), the layer-resolved Hall conductivities in the $x y$ plane is associated with the gradient of $\theta$, $\sigma_{x y}(z)=\frac{e^{2}}{2 \pi h} \partial_{z} \theta(z)$. Thus, the slab Hall conductance Eq. (8) is given by the difference of the $\theta$ values of bottom and top vacuum,

$$
\sigma_{x y}^{\text {slab }}=\int_{-\infty}^{\infty} d z \sigma_{x y}(z)=\frac{e^{2}}{h} \frac{\theta_{T}-\theta_{B}}{2 \pi},
$$

which is integerquantized independent of the $\theta$ value of the bulk. The gradient $\nabla \theta(\boldsymbol{r})$ is no vanishing at the interface between the two homogeneous materials with different $\theta$ values. Thus, the surface Hall conductances for the top and bottom interfaces are given by

$$
\begin{aligned}
\sigma_{x y}^{T} & =\frac{e^{2}}{h} \frac{\theta_{T}-\theta_{b}}{2 \pi}, \\
\sigma_{x y}^{B} & =\frac{e^{2}}{h} \frac{\theta_{b}-\theta_{B}}{2 \pi} .
\end{aligned}
$$

Apparently, the total slab Hall conductance is given by the summation of surface Hall conductances $\sigma_{x y}^{\text {slab }}=\sigma_{x y}^{T}+\sigma_{x y}^{B}$. Since the $\theta$ value for the top and bottom vacuum can only take the values $\theta_{T / B}=2 \pi n^{T / B}$ with $n^{T / B}$ being integer, for later convenience, we introduce the difference of the $\theta$ values between the top/bottom vacuum and the bulk of the material as

$$
2 \pi \tilde{n}_{z}^{T / B}=2 \pi n_{z}^{T / B}-\theta_{b} .
$$

As a result, the surface Hall conductance for the top and bottom interfaces can be expressed as $\sigma_{x y}^{T}=\frac{e^{2}}{h} \tilde{n}_{z}^{T}$ and $\sigma_{x y}^{B}=$ $-\frac{e^{2}}{h} \tilde{n}_{z}^{B}$, respectively.

\section{RELATION BETWEEN THE $\theta$ TERM AND THE CHIRAL HINGE MODES}

The current carried by the CHMs can be evaluated from the spatial dependent $\theta$, and each chiral hinge channel carries one conductance quantum $\left(e^{2} / h\right)$. We calculate the current through a $2 \mathrm{D}$ section disk $(D)$ encircling a hinge normal to the plane as illustrated in Fig. 2(a), $I=\iint_{D} d \boldsymbol{S} \cdot \boldsymbol{j}_{\theta}$. The electric field is determined by the gradient of a scalar potential, $\boldsymbol{E}=-\nabla \Phi(\boldsymbol{r})$, and we choose the boundary of the disk as an equipotential line $\Phi_{e}$. By utilizing Stokes theorem, $I=\frac{e^{2}}{2 \pi h} \oint_{C} d \boldsymbol{s} \cdot \nabla \theta(\boldsymbol{r}) \Phi(\boldsymbol{r})$. Thus, there is no current or equivalently gapless conducting channel on the hinge when $\theta(\mathbf{r})$ in the two vacuum areas takes the same value $n_{1}=n_{2}$. If they are different $n_{1} \neq n_{2}$, then there will be a branch cut separating the two vacuums where $\theta(\boldsymbol{r})$ is singular. In this situation, the contour integral gives the number of the conducting channels,

$$
\frac{I}{\Delta \Phi e^{2} / h}=n_{2}-n_{1}=\tilde{n}_{2}-\tilde{n}_{1},
$$

which is the winding number of the field $\theta(\boldsymbol{r}) . \Delta \Phi=\Phi_{e}-$ $\Phi_{\text {in }}$ denotes the potential difference between the outer contour $C_{\text {out }}$ and the inner contour $C_{\text {int }}$. In other words, the gapless hinge mode tracks the singularity of the $\theta$ term and vice versa. We also want to emphasize that, even when $\theta$ in the bulk is (a)

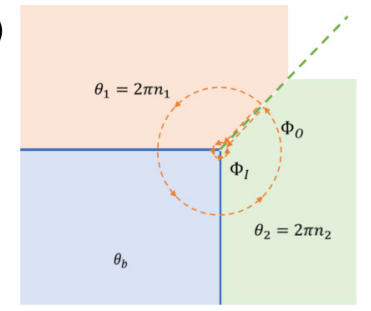

(c) 0

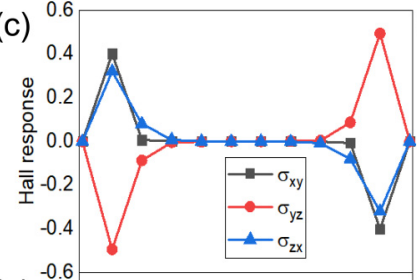

(e)

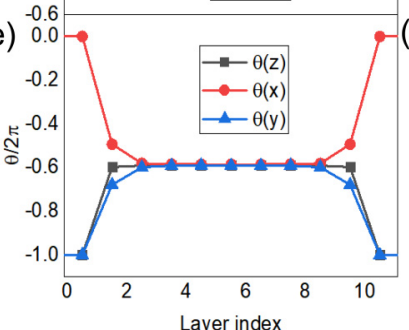

(b) $\theta(r, \varphi)$

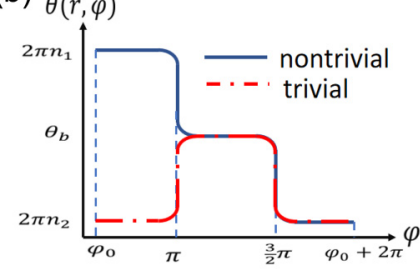

(d)

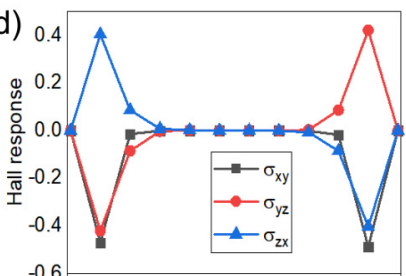

(f)

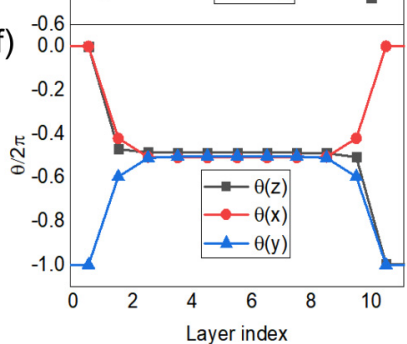

FIG. 2. (a) Schematic view of the hinge current. The planar surfaces of the topological insulator are characterized by integers $n_{1}$ and $n_{2}$, describing the integer change of the $\theta$ value nearby the surfaces. (b) Schematic view of $\theta$-term as a function of the angle $\varphi$ for topologically nontrivial and trivial cases. (c, d) Plots of the layer-resolved Hall response $\sigma_{\alpha \beta}\left(r_{\gamma}\right)$ and panels (e, f) are plots of the $\theta$-angle as function of the layer index for antiferromagnetic and rotoinversion quantum anomalous Hall insulator, respectively, from a layer-resolved Kubo formula in a slab geometry for 10 layers.

not quantized, the above argument for the gapless chiral hinge channel is still valid.

As show in Figs. 2(c)-2(f), we plot the layer-resolved Hall responses $\sigma_{\alpha \beta}\left(r_{\gamma}\right)\left(\epsilon_{\alpha \beta \gamma}=1\right)$ and the integrated value for $\theta\left(r_{\gamma}\right)$ as a function of the layer index for three directions. In numerical evaluation, we consider a slab geometry with the periodic boundary in the $\alpha \beta$ plane and open boundary condition in $r_{\gamma}$ direction. The layer resolved Hall response only distributes near the slab surfaces where $\theta$ changes and quickly drops to zero as the position moves into the bulk where $\theta$ takes constant value. For antiferromagnetic QAHI in Figs. 2(c) and $2(\mathrm{e})$, the magnetic point group $\mathcal{G}_{1}$ will put a constraint on the Hall response that the layer-resolved Hall conductivity takes the opposite values for the slab center. Thus, the slab Chern numbers vanish for three directions. Due to the presence of the mass term $d$, the axion angle will deviate form the quantized value $\pi$, for example, $\theta_{b} / 2 \pi \simeq-0.59$ in Fig. $2(\mathrm{e})$. It is also consistent with the symmetry analysis that there is no such symmetry to guarantee the quantization $\theta_{b}$ in $\widetilde{\mathcal{G}}_{1}$. As a consequence, the surface Hall conductance $\sigma_{x y}^{B}$ for the bottom interface and $\sigma_{x y}^{T}$ for the top interface are not half quantized in sharp contrast to the axion insulators. However, the summation of the surface Hall conductance of the adjacent surface must be quantized since $\sigma_{z x}^{i}+\sigma_{z y}^{j}=\frac{e^{2}}{h}\left(n_{y}^{i}-n_{x}^{j}\right)$ with $i, j=$ $T, B$, indicates whether the hinge mode at the intersection 
(a)

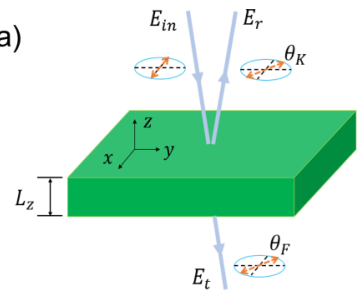

(b)

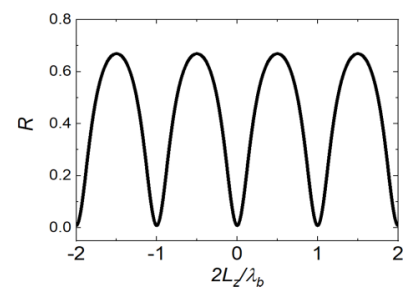

FIG. 3. (a) Schematic illustration of the measurement of Kerr and Faraday angle. Incident linearly polarized light becomes elliptically polarized after transmission (Faraday effect) and reflection (Kerr effect), with polarization angles as $\theta_{F}$ and $\theta_{K}$, respectively. (b) The reflectivity $R$ as a function of the slab thickness $L_{z}$ along $z$ direction in the units of half of photon wavelength $\lambda_{b} / 2$ for suspended single loop case with $\epsilon=10$ and $\mu=1$.

of two surfaces exists or not. For rotoinversion QAHI in Fig. 2(d), the symmetry $\widetilde{\mathcal{G}}_{2}$ constrains that the layer-resolved Hall conductivities for $z$ direction are symmetric about the slab center, while for $x$ and $y$ directions are antisymmetric about the slab center. The layer-resolved Hall conductivities in $x z$ plane and $y z$ plane are also related to each other by the $\mathcal{S}_{4}$ symmetry. Furthermore, as shown in Fig. 2(f), $\theta_{b}$ will be quantized due to the presence of improper rotation symmetry $\mathcal{S}_{4}$ and a combination of time-reversal and the diagonal mirror symmetry $\mathcal{T} \mathcal{M}^{x+y}$. As a result, the surface Hall conductance are half-quantized for three directions. More detailed discussion symmetry constraints on Hall response can be found in Appendix D. In this way, we establish the relation between the the CHMs and the two physical invariants,

$$
\begin{gathered}
\sigma_{\alpha \beta}^{\text {slab }}=\epsilon_{\alpha \beta \gamma} \frac{e^{2}}{h}\left(\tilde{n}_{\gamma}^{T}-\tilde{n}_{\gamma}^{B}\right), \\
\Delta_{\alpha \beta}=\delta_{\tilde{n}_{\beta}^{T}-\tilde{n}_{\beta}^{B}, 0} \delta_{\tilde{n}_{\alpha}^{T}-\tilde{n}_{\alpha}^{B}, 0}\left(\tilde{n}_{\alpha}^{T}-\tilde{n}_{\beta}^{T}\right),
\end{gathered}
$$

with $\epsilon_{\alpha \beta \gamma}=1$.

\section{MAGNETO-OPTICAL EFFECT AS A DETECTION OF TOPOLOGICAL INVARIANTS}

We propose an optical experiment to directly measure this phenomenon independent of material details as shown in Fig. 3(a). Consider a normally incident linearly x-polarized light with frequency $\omega$ propagating along the $z$ direction through the sample $\mathbf{E}_{\text {in }}=E_{\text {in }} \exp \left[i\left(k_{0} z-\omega t\right)\right] \hat{\mathbf{x}}$ with $k_{0}=$ $\omega / c . \mathbf{E}_{r}$ and $\mathbf{E}_{t}$ are the reflected and transmitted electric field, respectively. Their values at the interface between two materials are related to the incident field $\mathbf{E}_{\text {in }}$ by the $2 \times 2$ reflection and transmission tensors, and can be solved by matching the electrodynamic boundary conditions. The Kerr and Faraday angles are defined by the $\tan \theta_{K}=-E_{r}^{y} / E_{r}^{x}$ and $\tan \theta_{F}=E_{t}^{y} / E_{t}^{x}$, respectively [49,50]. When the chemical potential is located within the surface gap $E_{g}$ and $\hbar \omega \ll E_{g}$, the magnetic fields at the interface of the two materials are discontinuous due to the presence of surface Hall current. The reflection and transmission tensors for a slab can be obtained by composing the single-interface scattering matrices for top and bottom surfaces. For simplicity we only consider a freestanding sample, the influence of a substrate do not change our conclusion qualitatively. The reflectivity $R \equiv\left|\mathbf{E}_{r}\right|^{2} /\left|\mathbf{E}_{t}\right|^{2}$ will depend on the relative magnitude of the slab thickness and the wavelength $\left(\lambda_{b}=\frac{2 \pi c}{\omega \sqrt{\epsilon \mu}}\right)$ inside the bulk. As shown in Fig. 3(b), when the slab thickness contains an integer multiple of half wavelength $L_{z}=N \lambda_{b} / 2$ with an integer $N$ (the resonance condition), $R$ reaches the minima. At the resonance, the reflectivity can be obtained as

$$
R^{\prime}=\frac{\left[\alpha\left(\tilde{n}_{z}^{T}-\tilde{n}_{z}^{B}\right)\right]^{2}}{1+\left[\alpha\left(\tilde{n}_{z}^{T}-\tilde{n}_{z}^{B}\right)\right]^{2}}
$$

If the slab Chern number vanishes $\tilde{n}_{z}^{T}=\tilde{n}_{z}^{B} \neq 0$, then the light can propagate through the sample without reflection and the transmitted light is still linearly polarized in $\mathrm{x}$ direction. In this situation, the Faraday angle $\theta_{F}^{\prime}=0$. If the slab Chern number does not vanish, then the Faraday angle $\theta_{F}^{\prime}$ will be quantized in integer multiples of the fine structure constant $\alpha \equiv \frac{1}{4 \pi \epsilon_{0}} \frac{e^{2}}{\hbar c}$ [51],

$$
\tan \theta_{F}^{\prime}=\alpha\left(\tilde{n}_{z}^{T}-\tilde{n}_{z}^{B}\right),
$$

which is universal and independent of the specific value of $\theta_{b}$. As $\alpha \approx 1 / 137, \theta_{F}^{\prime} \approx \alpha\left(\tilde{n}_{z}^{T}-\tilde{n}_{z}^{B}\right)$. The slab Hall conductance Eq. (22) can thus be expressed in terms of the fundamental constants and the experimentally measurable quantity

$$
\sigma_{x y}^{\text {slab }}=\frac{e^{2}}{\alpha h} \tan \theta_{F}^{\prime}=\frac{e^{2}}{h}\left(\tilde{n}_{z}^{T}-\tilde{n}_{z}^{B}\right) .
$$

For the Kerr angle, we have

$$
\cot \theta_{K}^{\prime}=\alpha\left(\tilde{n}_{z}^{T}-\tilde{n}_{z}^{B}\right),
$$

For the slab Chern number that is not so large, $\theta_{K}^{\prime}=$ $\tan ^{-1}\left[1 / \alpha\left(\tilde{n}_{z}^{T}-\tilde{n}_{z}^{B}\right)\right] \approx \frac{\pi}{2}-\alpha\left(\tilde{n}_{z}^{T}-\tilde{n}_{z}^{B}\right)$, the polarization of the reflected light will exhibit a full-quarter rotation relative to the incident light [51]. To determine $\tilde{n}_{z}^{T}$ and $\tilde{n}_{z}^{B}$ for top and bottom surface, we also need to use the results at reflectivity maxima when $L_{z}=\left(N+\frac{1}{2}\right) \lambda_{b} / 2$.

The corresponding reflectivity is

$$
R^{\prime \prime}=1-\frac{4 \frac{\tilde{\epsilon}}{\tilde{\mu}}}{\left(\frac{\tilde{\epsilon}}{\tilde{\mu}}\right)^{2}+2 \frac{\tilde{\epsilon}}{\tilde{\mu}}\left[1-(2 \alpha)^{2} \tilde{n}_{z}^{T} \tilde{n}_{z}^{B}\right]+\left[1+\left(2 \alpha \tilde{n}_{z}^{T}\right)^{2}\right]\left[1+\left(2 \alpha \tilde{n}_{z}^{B}\right)^{2}\right]},
$$

with $\tilde{\epsilon}$ and $\tilde{\mu}$ being the relative permittivity and permeability for the material, respectively. For relative small slab Chern number $R^{\prime \prime} \approx\left(\frac{\tilde{\epsilon}}{\tilde{\tilde{\mu}}}-1\right)^{2} /\left(\frac{\tilde{\epsilon}}{\tilde{\mu}}+1\right)^{2}$ is only determined by the material constants. The corresponding Kerr $\theta_{K}^{\prime \prime}$ and Faraday 
angles $\theta_{F}^{\prime \prime}$ can be obtained as

$$
\begin{gathered}
\tan \theta_{K}^{\prime \prime} \\
=\frac{2\left\{\frac{\tilde{\epsilon}}{\tilde{\mu}} 2 \alpha\left(-\tilde{n}_{z}^{B}\right)-2 \alpha \tilde{n}_{z}^{T}\left[1+\left(2 \alpha \tilde{n}_{z}^{B}\right)^{2}\right]\right\}}{-\left(\frac{\tilde{\epsilon}}{\tilde{\mu}}\right)^{2}-2 \frac{\tilde{\epsilon}}{\tilde{\mu}} 2 \alpha \tilde{n}_{z}^{T} 2 \alpha \tilde{n}_{z}^{B}+\left[1-\left(2 \alpha \tilde{n}_{z}^{T}\right)^{2}\right]\left[1+\left(2 \alpha \tilde{n}_{z}^{B}\right)^{2}\right]}, \\
\tan \theta_{F}^{\prime \prime}=\frac{2 \alpha\left(\tilde{n}_{z}^{T}-\tilde{n}_{z}^{B}\right)}{1+\frac{\tilde{\epsilon}}{\tilde{\mu}}+2 \alpha \tilde{n}_{z}^{T} 2 \alpha \tilde{n}_{z}^{B}} .
\end{gathered}
$$

By canceling the explicit dependence on the materials properties $\tilde{\epsilon}$ and $\tilde{\mu}$, the measured Faraday angle $\theta_{F}^{\prime \prime}$ and Kerr angle $\theta_{K}^{\prime \prime}$ give a relation (see Appendix E)

$$
\tan \left(\theta_{K}^{\prime \prime}+\theta_{F}^{\prime \prime}\right)\left(1-\frac{\tan \theta_{F}^{\prime}}{\tan \theta_{F}^{\prime \prime}}\right)=\alpha\left(\tilde{n}_{z}^{T}+\tilde{n}_{z}^{B}\right) .
$$

Using the two relations in Eqs. (25) and (31), we can determine the values of $\tilde{n}_{z}^{T}$ and $\tilde{n}_{z}^{B}$ as

$$
\begin{aligned}
& \tilde{n}_{z}^{T}=\frac{1}{2 \alpha}\left[\tan \left(\theta_{K}^{\prime \prime}+\theta_{F}^{\prime \prime}\right)\left(1-\frac{\tan \theta_{F}^{\prime}}{\tan \theta_{F}^{\prime \prime}}\right)+\tan \theta_{F}^{\prime}\right], \\
& \tilde{n}_{z}^{B}=\frac{1}{2 \alpha}\left[\tan \left(\theta_{K}^{\prime \prime}+\theta_{F}^{\prime \prime}\right)\left(1-\frac{\tan \theta_{F}^{\prime}}{\tan \theta_{F}^{\prime \prime}}\right)-\tan \theta_{F}^{\prime}\right] .
\end{aligned}
$$

Similarly, $\tilde{n}_{x}^{T / B}$ and $\tilde{n}_{y}^{T / B}$ can be determined by the magnetooptical Faraday and Kerr measurements in the samples of thin films normal to other two crystallographic axes. Armed with all $\tilde{n}_{\alpha}^{T / B}(\alpha=x, y, z)$, one can determine the values of all the quadrupole indices [Eq. (23)] and the slab Chern numbers [Eq. (22)], and figure out the flowing pattern of chiral hinge currents [Eq. (21)].

\section{CONCLUSION}

In a 3D second-order topological insulator, the CHMs circulate along the hinges of a sample and lead to the $3 \mathrm{D}$ quantum anomalous Hall effect. The quadrupole index and the slab Chern numbers are introduced to characterize the flowing pattern of the CHMs. The nonzero slab Chern numbers determine the formation of the close path of the CHMs while the quadrupole index indicates the existence of CHMs along the four parallel hinges of a sample. The two topological invariants can be determined by the magneto-optical Faraday and Kerr measurements. The relation between the two topological invariants and the flowing pattern of the chiral hinge states reflects the bulk-boundary correspondence in 3D second-order topological insulators.

\section{ACKNOWLEDGMENTS}

B.F. and Z.A.H. contributed equally. This work was supported by the Research Grants Council, University Grants Committee, Hong Kong under Grant No. 17301220, and the National Key R\&D Program of China under Grant No. 2019YFA0308603.

\section{APPENDIX A: MODEL HAMILTONIAN, BAND STRUCTURE, AND THE WINDING OF QUADRUPOLE MOMENTS}

To facilitate numerical calculations, we map the continuous Hamiltonian model in the main text on a square lattice in the tight binding approximation, $k_{i} \rightarrow \frac{1}{a} \sin k_{i} a$ and $k_{i}^{2} \rightarrow \frac{2}{a^{2}}(1-$ $\cos k_{i} a$ ) for $i=x, y, z$. The lattice constant $a=1$ is chosen as the same for three directions. $\frac{1}{2}\left(i v_{\perp} \sigma_{x} s_{i}-2 m_{\perp} \sigma_{z} s_{0} \mp 2 c \sigma_{i} s_{0}\right)$ are the hopping matrices connecting the nearest neighbor sites along $x, y$ direction, respectively, and $\frac{1}{2}\left(i v_{z} \sigma_{x} s_{z}-2 m_{z} \sigma_{z} s_{0}\right)$ is the matrix connecting the next nearest neighbor sites along two directions in the $x-y$ plane, which encode the hopping between degrees of freedom between two sites. $\left(m_{0}+4 m_{\perp}+\right.$ $\left.2 m_{z}\right) \sigma_{z} s_{0}+d \sigma_{y} s_{0}+\sum_{i=x, y, z} b_{i} s_{i}$ are the on-site energies. In this representation, the Hamiltonian in momentum space is

$$
\mathcal{H}(\boldsymbol{k})=\mathcal{H}_{0}(\boldsymbol{k})+\sum_{i=1,2,3} \mathcal{V}_{i}(\boldsymbol{k})
$$

with

$$
\begin{aligned}
\mathcal{H}_{0}(\boldsymbol{k})= & \hbar \sigma_{x}\left[v_{\perp}\left(\sin k_{x} s_{x}+\sin k_{y} s_{y}\right)+v_{z} \sin k_{z} s_{z}\right] \\
& +\left[m_{0}+2 m_{\perp}\left(2-\cos k_{x}-\cos k_{y}\right)\right. \\
& \left.+2 m_{z}\left(1-\cos k_{z}\right)\right] \sigma_{z} s_{0}, \\
\mathcal{V}_{1}(\boldsymbol{k})= & -2 c\left(\cos k_{x}-\cos k_{y}\right) \sigma_{y} s_{0}, \\
\mathcal{V}_{2}(\boldsymbol{k})= & d \sigma_{y} s_{0}, \quad \mathcal{V}_{3}(\boldsymbol{k})=\sum_{i=x, y, z} b_{i} s_{i} .
\end{aligned}
$$

In the Hamiltonian Eq. (A1), $\mathcal{H}_{0}$ describes a 3D quantum spin Hall insulator. Throughout this work, we choose the parameters

$$
v_{z}=v_{\perp}=1, m_{z}=m_{\perp}=-0.5, m_{0}=0.5,
$$

to realize the topological nontrivial phase. To illustrate the differences of the hinge states between distinct higher-order topological phases, we calculate the band structure in a quasi-1D geometry along three different directions for the antiferromagnetic QAHI and the rotoinversion QAHI. To formulate these two cases, we need to add additional terms to the model of 3D quantum spin Hall system $\mathcal{H}_{0}$.

(i) To realize the rotoinversion QAHI, we include both $\mathcal{V}_{1}$ and $\mathcal{V}_{3}$ and use the parameters

$$
c=1, b_{z}=0.3, b_{x}=0, b_{y}=0 .
$$

In Figs. 4(a)-4(c), we show the band structures in the bar geometry along three directions. We can find two 1D chiral modes in $x$ and $y$ directions in the energy gap of the surface states which are singly degenerated with oppositely chiral modes localized on the neighboring two hinges. The rod bands along $z$ direction exhibit four chiral modes which are doubly degenerated and localized at four hinges with the same chiral modes on opposing sides. In Figs. 5(a)-5(c), we show the quadrupole moments for the two-dimensional subspace as functions of the momentum in the third direction. Only the quadrupole moment along the $z$ direction exhibits a nontrivial winding number which is consistent with the band calculations in Figs. 4(a)-4(c). 

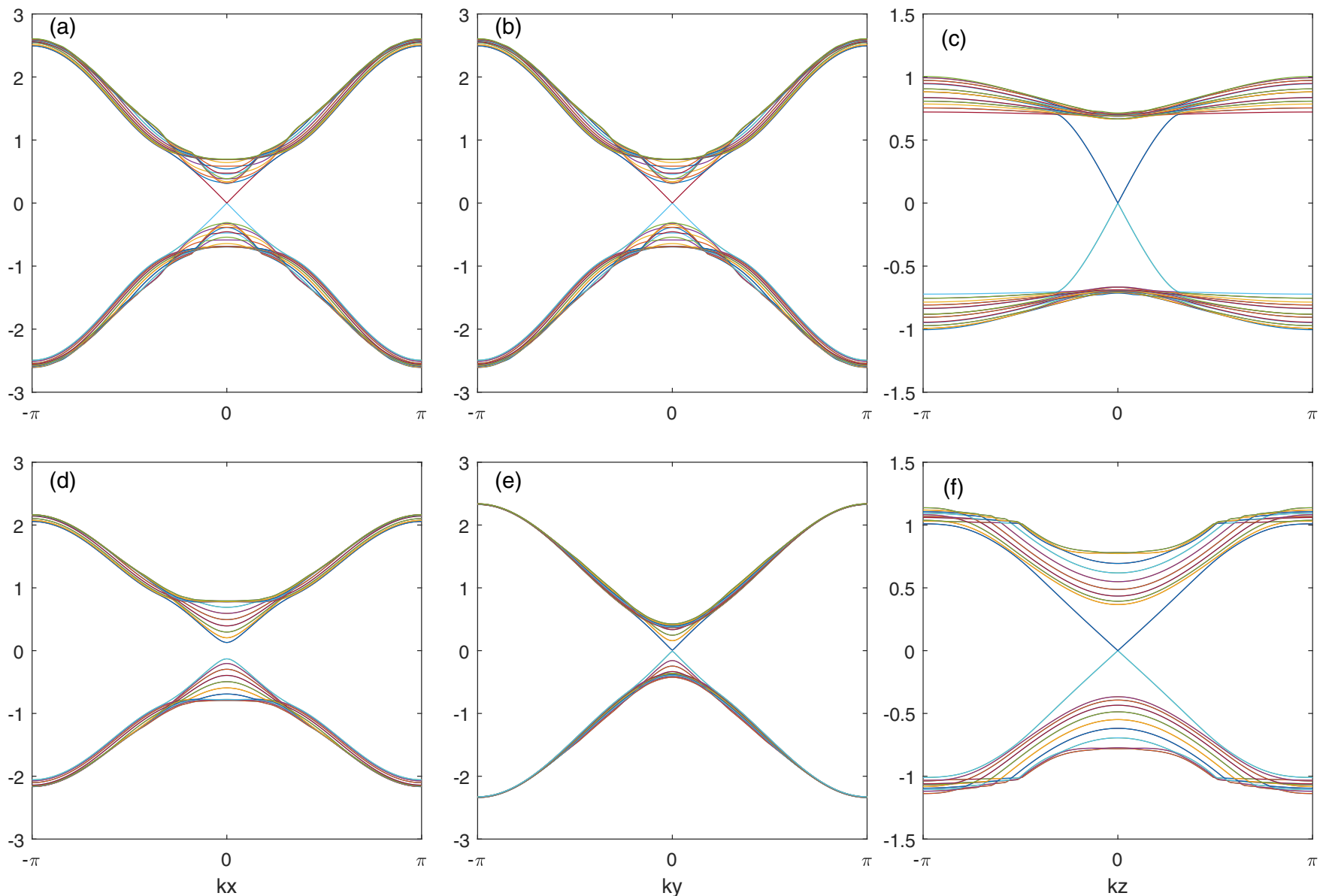

FIG. 4. The band structure of the rotoinversion QAHI and the antiferromagnetic QAHI in three directions. Panels (a-c) are the band structures of the 40 lowest bands along three directions of the rotoinversion QAHI. We use the parameters $c=1$ and $b_{z}=0.3, b_{x}=0, b_{y}=0$. Panels (d-f) are band structures of the antiferromagnetic QAHI along three directions. We use the parameters $d=0.2, c=1$. The parameters in $\mathcal{H}_{0}$ are same for two cases: $v_{z}=v_{\perp}=1, m_{z}=m_{\perp}=-0.5, m_{0}=0.5$.

(ii) To realize the antiferromagnetic QAHI, we include both $\mathcal{V}_{1}$ and $\mathcal{V}_{2}$ and use the parameters

$$
d=0.2, c=1 .
$$

In Figs. 4(d)-4(f), we show the band structures in the bar geometry along three directions. We can only find the chiral modes along $y$ and $z$ directions in the band gap. The in-gap chiral modes for two directions are all doubly degenerated and localized at four hinges. It is further confirmed by the winding of the quadrupole moments in Figs. 5(d)-5(f) that the quadrupole moments along both the $y$ and $z$ directions exhibit nontrivial winding. Only directions with four chiral hinge modes exhibit nontrivial winding number in quadrupole moments. In order to fully characterize the pattern of the hinge states, we also need to introduce the slab Chern number as discussed in the main text.

\section{APPENDIX B: SYMMETRY OF THE HAMILTONIAN}

The group $\widetilde{\mathcal{G}_{0}}$ of Eq. (1) in the main text is a HeeschSchubnikov magnetic group of type II; the inclusion of any term in $\mathcal{V}_{i=1,2,3}$ breaks time reversal symmetry and several spatial symmetries and reduces it to a magnetic group of type III $\left(\widetilde{\mathcal{G}}^{\prime}, \widetilde{\mathcal{G}}_{i=1,2,3}\right.$ in the main text). In general, the reduced magnetic point group can be expressed as [39]

$$
\widetilde{\mathcal{G}_{i}}=N \oplus \mathcal{T}(G-N),
$$

where $G$ is a subgroup of $D_{4 h}$ and $N$ is a halving subgroup of $G$. The subgroups of $D_{4 h}$ has the following relations:

$$
D_{4 h} \supset\left\{\begin{array}{l}
D_{2 d} \supset S_{4} \Longrightarrow \widetilde{\mathcal{G}}^{\prime}, \widetilde{\mathcal{G}}_{2}, \\
D_{2 h} \supset D_{2} \Longrightarrow \widetilde{\mathcal{G}_{1}}, \\
C_{2 h} \supset C_{i} \Longrightarrow \widetilde{\mathcal{G}_{3}},
\end{array}\right.
$$

from which the reduced magnetic point group can be generated. The group $\widetilde{\mathcal{G}}=D_{4 h} \oplus \mathcal{T} D_{4 h}$ are given explicitly in Table I and the symmetry breaking due to the inclusion of $\mathcal{V}_{i=1,2,3}$ is also presented.

\section{APPENDIX C: HINGE STATE PATTERN AS COLORING THE FACE}

Identifying all possible chiral hinge patterns on a cuboid geometry is equivalent to find all the distinct ways that a six-sided cuboid can be painted by using two different colors (each face in one color). The hinge currents can be viewed to flow along the boundary of different colored faces. Two colored cubics are not distinct if they are only up to a rotation. We list all distinct colored cuboid patterns in the Fig. 6. The 

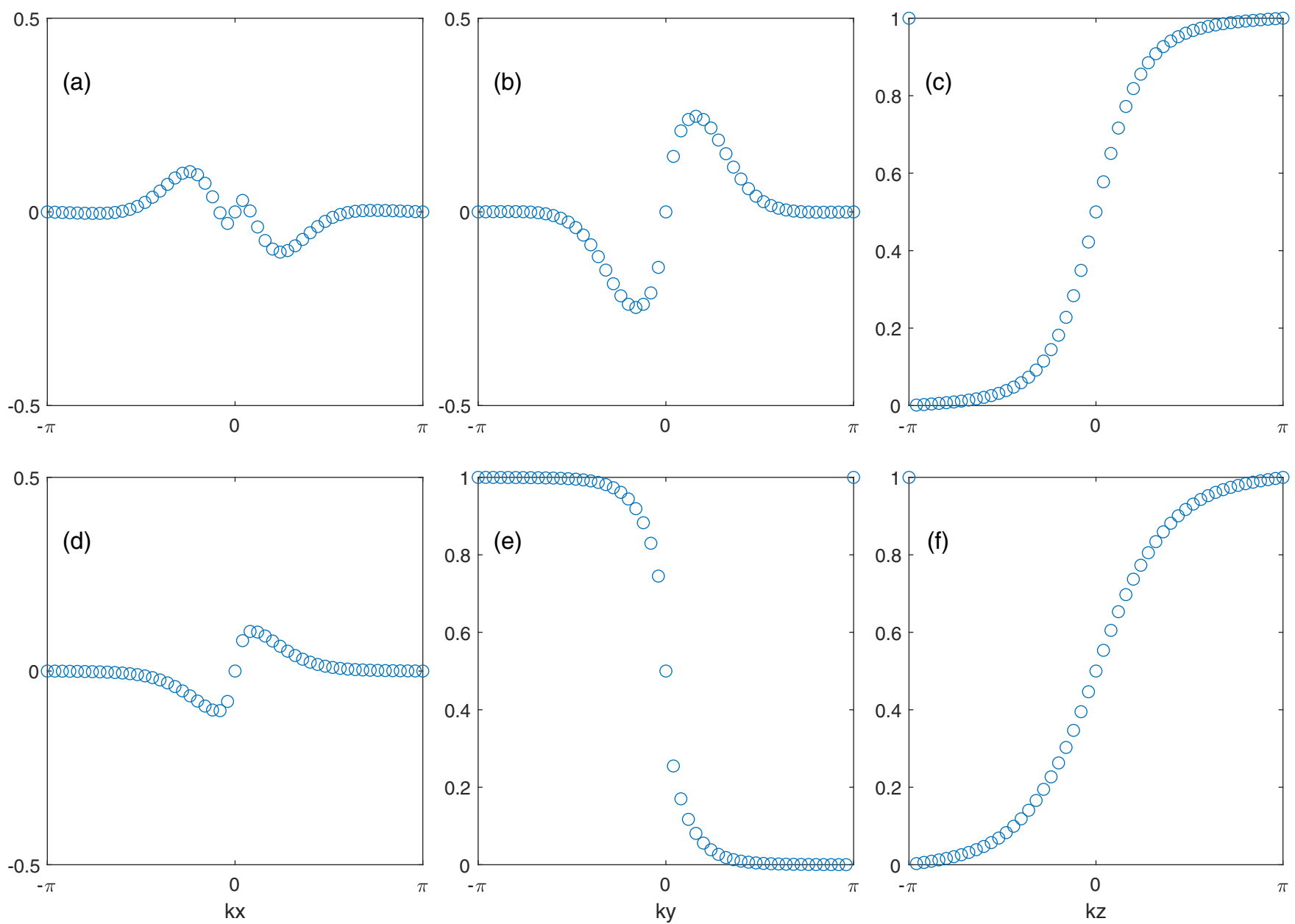

FIG. 5. The winding of quadrupole moments for the rotoinversion QAHI (a-c) and the antiferromagnetic QAHI (d-f) along three directions. The parameters are the same as described in the caption of Fig. 4.

first line in Fig. 6 lists all the distinct patterns that one of the faces is painted gray and all others white. The second line lists the patterns that two faces are gray and all others white. And the third line lists the patterns that three faces are gray.

All this results can be uniquely determined by the quadruple index and slab Chern number in Table II.

\section{APPENDIX D: SYMMETRY CONSTRAINTS ON HALL RESPONSE}

The Bloch Hamiltonian $\mathcal{H}(\boldsymbol{\xi})$ respects the symmetry $\mathcal{O}$, i.e., $\mathcal{O} \mathcal{H}(\boldsymbol{\xi}) \mathcal{O}^{-1}=\mathcal{H}\left(D_{\mathcal{O}} \xi\right)$, where $\boldsymbol{\xi}=\left(k_{\alpha}, k_{\beta}, r_{\gamma}\right)$ with $\alpha, \beta, \gamma$ representing three orthogonal directions. As shown in the second column of Table I, the unitary symmetry $\mathcal{O}_{u}=$ $U$ is a unitary matrix that acts on the internal degrees of freedom of the unit cell. The antiunitary symmetry $\mathcal{O}_{a}$ can be written as $\mathcal{O}_{a}=U \mathcal{K}$, where $U$ is a unitary operator and $\mathcal{K}$ is the complex conjugate operator. Due to the symmetry, the eigenstates of $\mathcal{H}(\xi)$ at $\xi$ and $D_{\mathcal{O}} \xi$ can be related by a gauge transformation. Explicitly, we can write the relations, $\mathcal{H}\left(D_{\mathcal{O}} \xi\right) \mathcal{O}\left|u_{n}(\xi)\right\rangle=\mathcal{O H}(\xi)\left|u_{n}(\xi)\right\rangle=\varepsilon_{n}(\xi) \mathcal{O}\left|u_{n}(\xi)\right\rangle$, for any eigenstate $\left|u_{n}(\xi)\right\rangle$ of $\mathcal{H}(\xi)$ with eigenvalue $\varepsilon_{n}(\xi)$ (the eigenvalue only depends on the momentum components). Thus, $\mathcal{O}\left|u_{n}(\xi)\right\rangle$ is an eigenstate of $\mathcal{H}\left(D_{\mathcal{O}} \xi\right)$ with the same energy. We can thus expand $\mathcal{O}\left|u_{n}(\xi)\right\rangle$ by the states at $D_{\mathcal{O}} \xi$,
$\mathcal{O}\left|u_{n}(\xi)\right\rangle=\sum_{m} B_{n, m}(\xi)\left|u_{m}\left(D_{\mathcal{O}} \xi\right)\right\rangle$ with $B_{n, m}(\xi)$ are the matrix elements of a unitary transformation acting on the space of the occupied bands. From which one can verify, for an antiunitary symmetry operator $\mathcal{O}_{a}$ and unitary symmetry operator $\mathcal{O}_{u}$, the Berry curvature satisfies

$$
\begin{aligned}
& \mathcal{F}_{\alpha \beta}(\xi)=-J_{\alpha \delta}^{\mathcal{O}_{a}} J_{\beta \zeta}^{\mathcal{O}_{a}} B(\xi) \mathcal{F}_{\delta \zeta}^{T}\left(D_{\mathcal{O}_{a}} \xi\right) B^{\dagger}(\xi), \\
& \mathcal{F}_{\alpha \beta}(\xi)=J_{\alpha \delta}^{\mathcal{O}_{u}} J_{\beta \zeta}^{\mathcal{O}_{u}} B^{*}(\xi) \mathcal{F}_{\delta \zeta}\left(D_{\mathcal{O}_{u}} \xi\right) B^{T}(\xi),
\end{aligned}
$$

respectively, with $J_{\alpha \beta}^{\mathcal{O}}=\partial\left(D_{\mathcal{O}} \boldsymbol{k}\right)_{\beta} / \partial \boldsymbol{k}_{\alpha}$. From Eq. (8) in the main text, the layered-resolved Hall responses have the following relations:

$$
\begin{aligned}
& \sigma_{\alpha \beta}\left(r_{\gamma}\right)=-J_{\alpha \delta}^{\mathcal{O}_{a}} J_{\beta \zeta}^{\mathcal{O}_{a}} \sigma_{\delta \zeta}\left[\left(D_{\mathcal{O}_{a}} \xi\right)_{\chi}\right], \\
& \sigma_{\alpha \beta}\left(r_{\gamma}\right)=J_{\alpha \delta}^{\mathcal{O}_{u}} J_{\beta \zeta}^{\mathcal{O}_{u}} \sigma_{\delta \zeta}\left[\left(D_{\mathcal{O}_{u}} \xi\right)_{\chi}\right]
\end{aligned}
$$

where $\delta, \zeta, \chi$ are another three orthogonal directions. The constraints on the Hall response due to the certain symmetry for three different higher-order topological phases are presented in Table III. 
TABLE I. Symmetry of the Hamiltonian $\mathcal{H}_{0}\left(k_{x}, k_{y}, k_{z}\right)$, on lines: time-reversal antiunitary symmetry $(\mathcal{T})$, chiral antisymmetry $(\mathcal{C}=\mathcal{P} \mathcal{T})$, particle-hole antiunitary antisymmetry $(\mathcal{P})$, fourfold rotation (around $z$ axis, $\mathcal{R}_{4}^{z}$ ), twofold rotation (around $j$ axis, $\mathcal{R}_{2}^{j}$ ), inversion symmetry $(\mathcal{I})$, fourfold improper rotation (around $z$ axis, $\left.\mathcal{S}_{4}\right)$, mirror reflection $\left(\mathcal{M}_{j}\right.$ the mirror plane is normal to $j$ axis), and the antiunitary symmetries (the combination of time reversal symmetry $\mathcal{T}$ with the above spatial symmetries). For each symmetry, the operator of transformation is given in the second column and the momentum is transformed according to the third column. The forth column: the Jacobian of the transformation. The last five columns indicate which symmetry is further broken with the inclusion of $\mathcal{V}_{i=1,2,3}$.

\begin{tabular}{|c|c|c|c|c|c|c|c|c|}
\hline Symmetries & Operators & $D_{\mathcal{O}}\left(k_{x}, k_{y}, k_{z}\right)$ & $\operatorname{det} J$ & $d \sigma_{y} s_{0}$ & $c\left(k_{x}^{2}-k_{y}^{2}\right) \sigma_{y} s_{0}$ & $b_{z} s_{z}$ & $b_{x} s_{x}$ & $b_{y} s_{y}$ \\
\hline $\mathcal{T}$ & $i s_{y} \mathcal{K}$ & $\left(-k_{x},-k_{y},-k_{z}\right)$ & -1 & $\chi$ & $\chi$ & $\chi$ & $\chi$ & $\chi$ \\
\hline $\mathcal{C}$ & $\sigma_{y}$ & $\left(k_{x}, k_{y}, k_{z}\right)$ & 1 & $\chi$ & $\chi$ & $\chi$ & $\chi$ & $\chi$ \\
\hline $\mathcal{P}=\mathcal{C} \mathcal{T}^{-1}$ & $-i \sigma_{y} s_{y} \mathcal{K}$ & $\left(-k_{x},-k_{y},-k_{z}\right)$ & -1 & $\sqrt{ }$ & $\sqrt{ }$ & $\sqrt{ }$ & $\sqrt{ }$ & $\sqrt{ }$ \\
\hline $\mathcal{R}_{4}^{z}\left(\mathcal{R}_{4}^{z-1}\right)$ & $\frac{\sqrt{2}}{2}\left(s_{0}+i s_{z}\right)$ & $\left(k_{y},-k_{x}, k_{z}\right)$ & 1 & $\sqrt{ }$ & $\chi$ & $\sqrt{ }$ & $\chi$ & $\chi$ \\
\hline $\mathcal{R}_{2}^{z}$ & $i s_{z}$ & $\left(-k_{x},-k_{y}, k_{z}\right)$ & 1 & $\sqrt{ }$ & $\sqrt{ }$ & $\sqrt{ }$ & $\chi$ & $\chi$ \\
\hline $\mathcal{R}_{2}^{x}\left(\mathcal{R}_{2}^{y}\right)$ & $i s_{x}$ & $\left(k_{x},-k_{y},-k_{z}\right)$ & 1 & $\sqrt{ }$ & $\sqrt{ }$ & $\chi$ & $\sqrt{ }(\chi)$ & $\chi(\sqrt{ })$ \\
\hline $\mathcal{R}_{2}^{x+y}\left(\mathcal{R}_{2}^{x-y}\right)$ & $\frac{\sqrt{2}}{2} i\left(s_{x}+s_{y}\right)$ & $\left(k_{y}, k_{x},-k_{z}\right)$ & 1 & $\sqrt{ }$ & $\chi$ & $\chi$ & $\chi$ & $\chi$ \\
\hline $\mathcal{I}$ & $\sigma_{z}$ & $\left(-k_{x},-k_{y},-k_{z}\right)$ & -1 & $\chi$ & $\chi$ & $\sqrt{ }$ & $\sqrt{ }$ & $\sqrt{ }$ \\
\hline $\mathcal{S}_{4}\left(\mathcal{S}_{4}^{-1}\right)$ & $\frac{\sqrt{2}}{2} \sigma_{z}\left(s_{0}+i s_{z}\right)$ & $\left(-k_{y}, k_{x},-k_{z}\right)$ & -1 & $\chi$ & $\sqrt{ }$ & $\sqrt{ }$ & $\chi$ & $\chi$ \\
\hline $\mathcal{M}_{z}$ & $i \sigma_{z} s_{z}$ & $\left(k_{x}, k_{y},-k_{z}\right)$ & -1 & $\chi$ & $\chi$ & $\sqrt{ }$ & $\chi$ & $\chi$ \\
\hline $\mathcal{M}_{x}\left(\mathcal{M}_{y}\right)$ & $i \sigma_{z} s_{x}$ & $\left(-k_{x}, k_{y}, k_{z}\right)$ & -1 & $\chi$ & $\chi$ & $\chi$ & $\sqrt{ }(\chi)$ & $\chi(\sqrt{ })$ \\
\hline $\mathcal{M}_{x+y}\left(\mathcal{M}_{x-y}\right)$ & $\frac{\sqrt{2}}{2} i \sigma_{z}\left(s_{x}+s_{y}\right)$ & $\left(-k_{y},-k_{x}, k_{z}\right)$ & -1 & $\chi$ & $\sqrt{ }$ & $\chi$ & $\chi$ & $\chi$ \\
\hline $\mathcal{T} \mathcal{R}_{4}^{z}\left(\mathcal{R}_{4}^{z-1}\right)$ & $\frac{\sqrt{2}}{2} i\left(s_{y}+s_{x}\right) \mathcal{K}$ & $\left(-k_{y}, k_{x},-k_{z}\right)$ & -1 & $\chi$ & $\sqrt{ }$ & $\chi$ & $\sqrt{ }$ & $\sqrt{ }$ \\
\hline $\mathcal{T} \mathcal{R}_{2}^{z}$ & $i s_{x} \mathcal{K}$ & $\left(k_{x}, k_{y},-k_{z}\right)$ & -1 & $\chi$ & $\chi$ & $\chi$ & $\sqrt{ }$ & $\sqrt{ }$ \\
\hline $\mathcal{T} \mathcal{R}_{2}^{x}\left(\mathcal{R}_{2}^{y}\right)$ & $-i s_{z} \mathcal{K}$ & $\left(-k_{x}, k_{y}, k_{z}\right)$ & -1 & $\chi$ & $\chi$ & $\sqrt{ }$ & $\chi(\sqrt{ })$ & $\sqrt{ }(\chi)$ \\
\hline $\mathcal{T} \mathcal{R}_{2}^{x+y}\left(\mathcal{R}_{2}^{x-y}\right)$ & $-\frac{\sqrt{2}}{2}\left(s_{0}+i s_{z}\right) \mathcal{K}$ & $\left(-k_{y},-k_{x}, k_{z}\right)$ & -1 & $\chi$ & $\sqrt{ }$ & $\sqrt{ }$ & $\sqrt{ }$ & $\sqrt{ }$ \\
\hline $\mathcal{T I}$ & $\sigma_{z} i s_{y} \mathcal{K}$ & $\left(k_{x}, k_{y}, k_{z}\right)$ & 1 & $\sqrt{ }$ & $\sqrt{ }$ & $\chi$ & $\chi$ & $\chi$ \\
\hline $\mathcal{T} \mathcal{S}_{4}\left(\mathcal{S}_{4}^{-1}\right)$ & $\frac{\sqrt{2}}{2} i \sigma_{z}\left(s_{y}+s_{x}\right) \mathcal{K}$ & $\left(k_{y},-k_{x}, k_{z}\right)$ & 1 & $\sqrt{ }$ & $\chi$ & $\chi$ & $\sqrt{ }$ & $\sqrt{ }$ \\
\hline $\mathcal{T} \mathcal{M}_{z}$ & $i \sigma_{z} s_{x} \mathcal{K}$ & $\left(-k_{x},-k_{y}, k_{z}\right)$ & 1 & $\sqrt{ }$ & $\sqrt{ }$ & $\chi$ & $\sqrt{ }$ & $\sqrt{ }$ \\
\hline $\mathcal{T} \mathcal{M}_{x}\left(\mathcal{M}_{y}\right)$ & $-i \sigma_{z} s_{z} \mathcal{K}$ & $\left(k_{x},-k_{y},-k_{z}\right)$ & 1 & $\sqrt{ }$ & $\sqrt{ }$ & $\sqrt{ }$ & $\chi(\sqrt{ })$ & $\sqrt{ }(\chi)$ \\
\hline $\mathcal{T} \mathcal{M}_{x+y}\left(\mathcal{M}_{x-y}\right)$ & $-\frac{\sqrt{2}}{2}\left(s_{0}+i s_{z}\right) \sigma_{z} \mathcal{K}$ & $\left(k_{y}, k_{x},-k_{z}\right)$ & 1 & $\sqrt{ }$ & $\chi$ & $\sqrt{ }$ & $\sqrt{ }$ & $\sqrt{ }$ \\
\hline
\end{tabular}

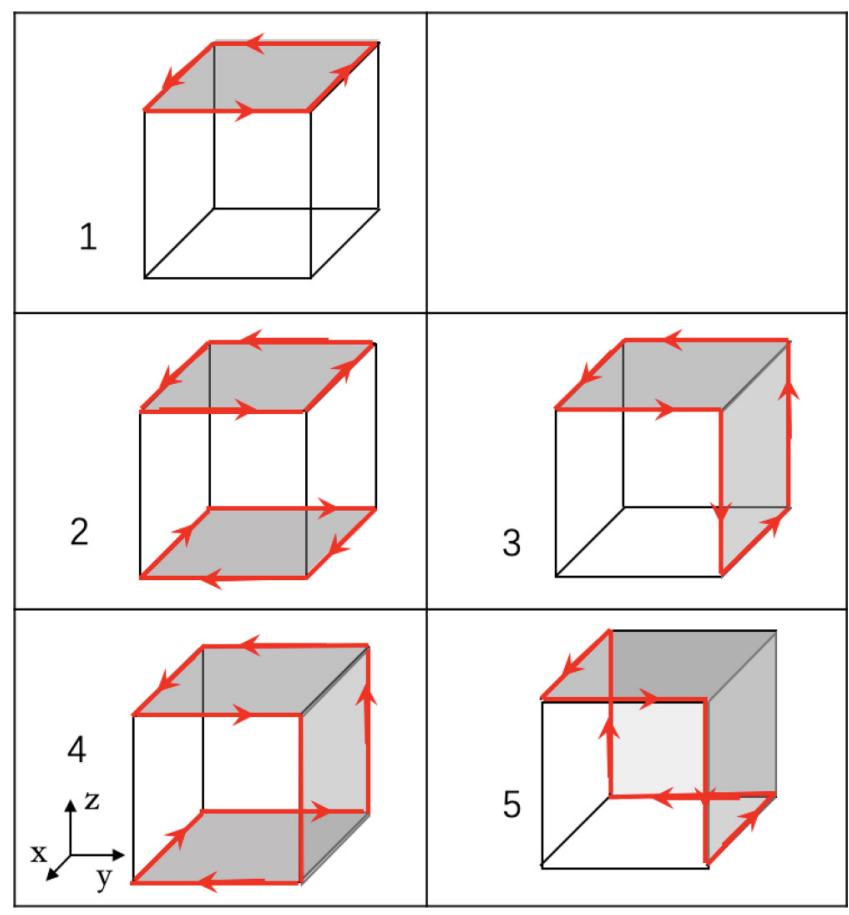

FIG. 6. All possible patterns of chiral hinge modes as the boundary of colored face on a cubic.

\section{APPENDIX E: MAGNETO-OPTICAL FARADAY AND KERR EFFECTS}

In the main text we propose an optical experiment to measure the topological invariants. We consider a suspended sample with a slab geometry of thickness $L_{z}$. A similar approach was considered in Ref. [50], but for the sake of completeness, we present the detailed derivations here. We first solve the reflection and transmission coefficients for the interface between two materials. Consider a light wave propagating along the $z$ direction through two materials, labeled by $i$ and $j$ with dielectric constant and magnetic permeability as $\epsilon_{i}, \mu_{i}$ and $\epsilon_{j}, \mu_{j}$, respectively. The interface is

TABLE II. The corresponding quadrupole index and slab Chern number of all pattern in Fig. 6 .

\begin{tabular}{cccccc}
\hline \hline & 1 & 2 & 3 & 4 & 5 \\
\hline$n_{x}$ & 0 & 0 & 0 & 0 & -1 \\
$n_{y}$ & 0 & 0 & 1 & 1 & 1 \\
$n_{z}$ & 1 & 0 & 1 & 0 & 1 \\
$\Delta_{x}$ & 0 & -1 & 0 & 0 & 0 \\
$\Delta_{y}$ & 0 & 1 & 0 & 1 & 0 \\
$\Delta_{z}$ & 0 & 0 & 0 & 0 & 0 \\
\hline \hline
\end{tabular}


TABLE III. The constraints on the Hall response due to the certain symmetry for three cases discussed in the main text according to Eqs. (D1). The symmetry constraints on the Hall response for each reduced magnetic point group are listed in the last line for each topological phase.

\begin{tabular}{lc}
\hline \hline$D_{2} \oplus \mathcal{T}\left(D_{2 h}-D_{2}\right)$ & Antiferromagnetic QAHI \\
\hline $\mathcal{R}_{2}^{z}$ & $\sigma_{x z}(y)=-\sigma_{x z}(-y), \sigma_{y z}(x)=-\sigma_{y z}(-x)$ \\
$\mathcal{R}_{2}^{x}$ & $\sigma_{x y}(z)=-\sigma_{x y}(-z), \sigma_{x z}(y)=-\sigma_{x z}(-y)$ \\
$\mathcal{T} \mathcal{I}$ & $\sigma_{x y}(z)=-\sigma_{x y}(-z)$ \\
$\mathcal{T} \mathcal{M}_{z}$ & $\sigma_{y z}(x)=-\sigma_{y z}(-x)$ \\
$\mathcal{T} \mathcal{M}_{x}$ & $\sigma_{x y}(z)=-\sigma_{x y}(-z), \sigma_{x z}(y)=-\sigma_{y z}(-x)$ \\
$\widetilde{\mathcal{G}}_{1}$ & $\sigma_{x y}(z)=-\sigma_{x y}(-z), \sigma_{x z}(y)=-\sigma_{x z}(-y), \sigma_{y z}(x)=-\sigma_{y z}(-x)$ \\
$S_{4} \oplus \mathcal{T}\left(D_{2 d}-S_{4}\right)$ & $\operatorname{Rotoinversion~QAHI}$ \\
$\mathcal{R}_{2}^{z}$ & $\sigma_{x z}(y)=-\sigma_{x z}(-y), \sigma_{y z}(x)=-\sigma_{y z}(-x)$ \\
$\mathcal{S}_{4}$ & $\sigma_{x y}(z)=-\sigma_{y x}(-z), \sigma_{x z}(y)=\sigma_{y z}(x), \sigma_{y z}(x)=-\sigma_{x z}(-y)$ \\
$\mathcal{T} \mathcal{R}_{2}^{x+y}$ & $\sigma_{x y}(z)=-\sigma_{y x}(-z), \sigma_{x z}(y)=\sigma_{y z}(x), \sigma_{y z}(x)=\sigma_{x z}(y)$ \\
$\mathcal{T} \mathcal{M}_{x}$ & $\sigma_{y z}(x)=-\sigma_{y z}(-x)$ \\
$\widetilde{\mathcal{G}}_{2}$ & $\sigma_{x z}(y)=-\sigma_{x z}(-y)=\sigma_{y z}(x)=-\sigma_{y z}(-x), \sigma_{x y}(z)=\sigma_{x y}(-z)$ \\
$C_{i} \oplus \mathcal{T}\left(C_{2 h}-C_{i}\right)$ & $\operatorname{Inversion} \mathrm{QAHI}$ \\
\hline $\mathcal{I}$ & $\sigma_{x y}(z)=\sigma_{x y}(-z), \sigma_{z x}(y)=\sigma_{z x}(-y), \sigma_{y z}(x)=\sigma_{y z}(-x)$ \\
$\mathcal{T} \mathcal{R}_{2}^{x+y}$ & $\sigma_{x y}(z)=-\sigma_{y x}(-z), \sigma_{y z}(x)=\sigma_{x z}(y), \sigma_{z x}(y)=\sigma_{z y}(x)$ \\
$\mathcal{T} \mathcal{M}^{x+y}$ & $\sigma_{x y}(z)=-\sigma_{y x}(z), \sigma_{y z}(x)=\sigma_{x z}(-y), \sigma_{z x}(y)=\sigma_{z y}(-x)$ \\
$\widetilde{\mathcal{G}}_{3}$ & $\sigma_{x y}(z)=\sigma_{x y}(-z), \sigma_{z x}(y)=\sigma_{z x}(-y)=\sigma_{z y}(x)=\sigma_{z y}(-x)$ \\
\hline \hline
\end{tabular}

located at $z=z_{0}$. The electric field in the medium $i$ can be written as

$$
\mathbf{E}_{i}=e^{i k_{i} z} \mathbf{E}_{i}^{t}+e^{-i k_{i} z} \mathbf{E}_{i}^{r},
$$

where $\mathbf{E}_{i}^{r(t)}=\left[E_{i x}^{r(t)}, E_{i y}^{r(t)}\right]^{T}$ denote reflected (transmitted) components of the electric fields in the medium $i, k_{i}=$ $\frac{\omega}{c} \sqrt{\epsilon_{i} \mu_{i}}$ is the wave vector. The magnetic field is given by Faraday's law,

$$
\mathbf{H}_{i}=\sqrt{\frac{\epsilon_{i}}{\mu_{i}}}\left(-i \tau_{y}\right)\left\{e^{i k_{i} z} \mathbf{E}_{i}^{t}-e^{-i k_{i} z} \mathbf{E}_{i}^{r}\right\},
$$

where $\tau_{y}=\left[\begin{array}{cc}0 & -i \\ i & 0\end{array}\right]$ is the Pauli matrix acting on $x y$ space. The incoming and outgoing fields at the interface are related by the S matrix:

$$
\left[\begin{array}{l}
\mathbf{E}_{r}^{i} \\
\mathbf{E}_{t}^{j}
\end{array}\right]=\left[\begin{array}{ll}
\mathbf{r} & \mathbf{t}^{\prime} \\
\mathbf{t} & \mathbf{r}^{\prime}
\end{array}\right]\left[\begin{array}{l}
\mathbf{E}_{t}^{i} \\
\mathbf{E}_{r}^{j}
\end{array}\right],
$$

where $\mathbf{r}, \mathbf{r}^{\prime}$ and $\mathbf{t}, \mathbf{t}^{\prime}$ are all $2 \times 2$ reflection and transmission tensors. The electric field Eq. (E1) and magnetic field Eq. (E2) in the medium $i$ written in terms of the reflected and transmitted components of electric field is

$$
\left[\begin{array}{c}
\mathbf{E}_{i} \\
\mathbf{H}_{i}
\end{array}\right]=\left[\begin{array}{cc}
e^{i k_{i} z} & e^{-i k_{i} z} \\
\sqrt{\frac{\epsilon_{i}}{\mu_{i}}} e^{i k_{i} z}\left(-i \tau_{y}\right) & -\sqrt{\frac{\epsilon_{i}}{\mu_{i}}} e^{-i k_{i} z}\left(-i \tau_{y}\right)
\end{array}\right]\left[\begin{array}{c}
\mathbf{E}_{i}^{t} \\
\mathbf{E}_{j}^{t}
\end{array}\right] .
$$

Now consider the interface conditions for the electromagnetic fields, the electric field is continuous across the interface

$$
\left.\left(\mathbf{E}_{i}-\mathbf{E}_{j}\right)\right|_{z=z_{0}}=0,
$$

and the magnetic field will be discontinuous across the interface due to the presence of the surface current

$$
-\left.i \tau_{y}\left(\mathbf{H}_{j}-\mathbf{H}_{i}\right)\right|_{z=z_{0}}=\left.4 \pi \mathbf{J}_{i}\right|_{z=z_{0}},
$$

with the surface current density

$$
\left.\mathbf{J}_{i}\right|_{z=z_{0}}=\left.\boldsymbol{\sigma}_{i} \mathbf{E}_{i}\right|_{z=z_{0}},
$$

where $\sigma$ is the $2 \times 2$ conductivity tensor. The electromagnetic boundary conditions Eqs. (E5) and (E6) can be recast into the matrix form,

$$
\left.\left(\left[\begin{array}{c}
\mathbf{E}_{i} \\
\mathbf{H}_{i}
\end{array}\right]-\left[\begin{array}{c}
\mathbf{E}_{j} \\
\mathbf{H}_{j}
\end{array}\right]\right)\right|_{z=z_{0}}=-\left.4 \pi\left[\begin{array}{cc}
0 & 0 \\
i \tau_{y} \boldsymbol{\sigma}_{i} e^{i k_{i} z_{0}} & i \tau_{y} \boldsymbol{\sigma}_{i} e^{-i k_{i} z_{0}}
\end{array}\right]\left[\begin{array}{c}
\mathbf{E}_{i}^{t} \\
\mathbf{E}_{j}^{t}
\end{array}\right]\right|_{z=z_{0}} .
$$

Using Eq. (E4) and comparing with Eq. (E3), we directly obtain

$$
\begin{gathered}
\mathbf{r}=e^{2 i k_{i} z_{0}} \frac{\left[\frac{\epsilon_{i}}{\mu_{i}}-\left(\sqrt{\frac{\epsilon_{j}}{\mu_{j}}}+4 \pi \sigma_{x x}\right)^{2}-\left(4 \pi \sigma_{x y}\right)^{2}\right] \tau_{0}-\sqrt{\frac{\epsilon_{i}}{\mu_{i}}} 8 \pi \sigma_{x y} i \tau_{y}}{\left(\sqrt{\frac{\epsilon_{j}}{\mu_{j}}}+\sqrt{\frac{\epsilon_{i}}{\mu_{i}}}+4 \pi \sigma_{x x}\right)^{2}+\left(4 \pi \sigma_{x y}\right)^{2}}, \\
\mathbf{t}=e^{i\left(k_{i}-k_{j}\right) z_{0}} \frac{2 \sqrt{\frac{\epsilon_{i}}{\mu_{i}}}\left(\sqrt{\frac{\epsilon_{j}}{\mu_{j}}}+\sqrt{\frac{\epsilon_{i}}{\mu_{i}}}+4 \pi \sigma_{x x}\right) \tau_{0}-\sqrt{\frac{\epsilon_{i}}{\mu_{i}}} 8 \pi \sigma_{x y} i \tau_{y}}{\left(\sqrt{\frac{\epsilon_{j}}{\mu_{j}}}+\sqrt{\frac{\epsilon_{i}}{\mu_{i}}}+4 \pi \sigma_{x x}\right)^{2}+\left(4 \pi \sigma_{x y}\right)^{2}},
\end{gathered}
$$




$$
\begin{aligned}
& \mathbf{r}^{\prime}=e^{-2 i k_{j} z_{0}} \frac{\left[\frac{\epsilon_{j}}{\mu_{j}}-\left(\sqrt{\frac{\epsilon_{i}}{\mu_{i}}}+4 \pi \sigma_{x x}\right)^{2}-\left(4 \pi \sigma_{x y}\right)^{2}\right] \tau_{0}-\sqrt{\frac{\epsilon_{j}}{\mu_{j}}} 8 \pi \sigma_{x y} i \tau_{y}}{\left(\sqrt{\frac{\epsilon_{j}}{\mu_{j}}}+\sqrt{\frac{\epsilon_{i}}{\mu_{i}}}+4 \pi \sigma_{x x}\right)^{2}+\left(4 \pi \sigma_{x y}\right)^{2}}, \\
& \mathbf{t}^{\prime}=\frac{2 \sqrt{\frac{\epsilon_{j}}{\mu_{j}}}\left(\sqrt{\frac{\epsilon_{j}}{\mu_{j}}}+\sqrt{\frac{\epsilon_{i}}{\mu_{i}}}+4 \pi \sigma_{x x}\right) \tau_{0}-i \tau_{y} \sqrt{\frac{\epsilon_{j}}{\mu_{j}}} 8 \pi \sigma_{x y}}{\left(\sqrt{\frac{\epsilon_{j}}{\mu_{j}}}+\sqrt{\frac{\epsilon_{i}}{\mu_{i}}}+4 \pi \sigma_{x x}\right)^{2}+\left(4 \pi \sigma_{x y}\right)^{2}} .
\end{aligned}
$$

Thus, $\mathbf{r}^{\prime}$ is related to $\mathbf{r}$ by making the replacement $k_{i} \rightarrow-k_{j}$ and interchanging $\epsilon_{i} / \mu_{i}$ and $\epsilon_{j} / \mu_{j}$ and $\mathbf{t}^{\prime}$ can be obtained from $\mathbf{t}$ by interchanging $\epsilon_{i} / \mu_{i}$ and $\epsilon_{j} / \mu_{j}$. The reflection $\left(\mathbf{r}_{S}^{(\prime)}\right)$ and transmission tensor $\left(\mathbf{t}_{S}^{(/)}\right)$for a slab can be composed from the single-interface scattering matrices $\mathbf{r}_{T, B}^{(\prime)}$ and $\mathbf{t}_{T, B}^{(\prime)}$ for the top and bottom surfaces. We assume the two interfaces between the vacuum and the sample are at $z=0$ and $z=L_{z}$, respectively. The vacuum outside the sample has $\epsilon_{0}=\mu_{0}=1$. The electric fields at the top and bottom interface have the following relations:

$$
\begin{aligned}
& {\left[\begin{array}{c}
\mathbf{E}_{r}^{T} \\
\mathbf{E}_{t}^{I}
\end{array}\right]=\left[\begin{array}{ll}
\mathbf{r}_{T} & \mathbf{t}_{T}^{\prime} \\
\mathbf{t}_{T} & \mathbf{r}_{T}^{\prime}
\end{array}\right]\left[\begin{array}{c}
\mathbf{E}_{t}^{T} \\
\mathbf{E}_{r}^{I}
\end{array}\right],} \\
& {\left[\begin{array}{c}
\mathbf{E}_{r}^{I} \\
\mathbf{E}_{t}^{B}
\end{array}\right]=\left[\begin{array}{ll}
\mathbf{r}_{B} & \mathbf{t}_{B}^{\prime} \\
\mathbf{t}_{B} & \mathbf{r}_{B}^{\prime}
\end{array}\right]\left[\begin{array}{c}
\mathbf{E}_{t}^{I} \\
\mathbf{E}_{r}^{B}
\end{array}\right],}
\end{aligned}
$$

where $\mathbf{E}_{t}^{I}$ and $\mathbf{E}_{r}^{I}$ are the transmitted and reflected components of the electric fields in the sample, respectively. We also have

$$
\left[\begin{array}{l}
\mathbf{E}_{r}^{T} \\
\mathbf{E}_{t}^{B}
\end{array}\right]=\left[\begin{array}{ll}
\mathbf{r}_{S} & \mathbf{t}_{S}^{\prime} \\
\mathbf{t}_{S} & \mathbf{r}_{S}^{\prime}
\end{array}\right]\left[\begin{array}{l}
\mathbf{E}_{t}^{T} \\
\mathbf{E}_{r}^{B}
\end{array}\right] .
$$

Then we can obtain the reflection and transmission tensor for the slab

$$
\begin{aligned}
\mathbf{r}_{S} & =\mathbf{r}_{T}+\mathbf{t}_{T}^{\prime} \mathbf{r}_{B}\left(1-\mathbf{r}_{T}^{\prime} \mathbf{r}_{B}\right)^{-1} \mathbf{t}_{T}, \\
\mathbf{t}_{S} & =\mathbf{t}_{B}\left(1-\mathbf{r}_{T}^{\prime} \mathbf{r}_{B}\right)^{-1} \mathbf{t}_{T}, \\
\mathbf{t}_{S}^{\prime} & =\mathbf{t}_{T}^{\prime}\left(1-\mathbf{r}_{B} \mathbf{r}_{T}^{\prime}\right)^{-1} \mathbf{t}_{B}^{\prime}, \\
\mathbf{r}_{S}^{\prime} & =\mathbf{r}_{B}^{\prime}+\mathbf{t}_{B} \mathbf{r}_{T}^{\prime}\left(1-\mathbf{r}_{B} \mathbf{r}_{T}^{\prime}\right)^{-1} \mathbf{t}_{B}^{\prime} .
\end{aligned}
$$

For an incident light linearly polarized in the $x$ direction

$$
\mathbf{E}_{\text {in }}=E_{\text {in }} \hat{\mathbf{x}} \text {. }
$$

The reflectivity is defined as

$$
R \equiv \frac{\left|\mathbf{E}_{r}\right|^{2}}{\left|\mathbf{E}_{\mathrm{in}}\right|^{2}}=\frac{\left|r_{x x} E_{\mathrm{in}}\right|^{2}+\left|r_{x y} E_{\mathrm{in}}\right|^{2}}{\left|E_{\mathrm{in}}\right|^{2}}=\left|r_{x x}\right|^{2}+\left|r_{x y}\right|^{2},
$$

and the Kerr and Faraday angles are defined by

$$
\tan \theta_{K}=-\frac{E_{r}^{y}}{E_{r}^{x}}=-\frac{r_{y x} E_{\text {in }}}{r_{x x} E_{\text {in }}}=-\frac{r_{y x}}{r_{x x}}=\frac{r_{x y}}{r_{x x}},
$$

and

$$
\tan \theta_{F}=\frac{E_{t}^{y}}{E_{t}^{x}}=\frac{t_{y x} E_{\mathrm{in}}}{t_{x x} E_{\mathrm{in}}}=\frac{t_{y x}}{t_{x x}}=-\frac{t_{x y}}{t_{x x}},
$$

respectively. The reflectivity $R$ depends on the relative magnitude of the slab thickness and the wavelength inside the bulk. For the cavity resonance condition $k L_{z}=N \pi$ is satisfied where $k=\sqrt{\epsilon \mu} \omega / c$ is the wave number in the slab film and $N$ is an integer, we have

$$
\left\{\begin{array}{c}
r_{S, x x}^{\min } \\
r_{S, x y}^{\min }
\end{array}\right\}=\frac{1}{4 \frac{\epsilon_{0}}{\mu_{0}}+\left[4 \pi\left(\sigma_{x y}^{T}+\sigma_{x y}^{B}\right)\right]^{2}}\left\{\begin{array}{c}
-\left[4 \pi\left(\sigma_{x y}^{T}+\sigma_{x y}^{B}\right)\right]^{2} \\
-\sqrt{\frac{\epsilon_{0}}{\mu_{0}}} 8 \pi\left(\sigma_{x y}^{T}+\sigma_{x y}^{B}\right)
\end{array}\right\}
$$

and

$$
\left\{\begin{array}{l}
t_{S, x x}^{\min } \\
t_{S, x y}^{\min }
\end{array}\right\}=\frac{e^{-i k_{0} L_{z}} \sqrt{\frac{\epsilon_{0}}{\mu_{0}}}}{4 \frac{\epsilon_{0}}{\mu_{0}}+\left[4 \pi\left(\sigma_{x y}^{T}+\sigma_{x y}^{B}\right)\right]^{2}}\left\{\begin{array}{c}
-4 \sqrt{\frac{\epsilon_{0}}{\mu_{0}}} \\
8 \pi\left(\sigma_{x y}^{T}+\sigma_{x y}^{B}\right)
\end{array}\right\},
$$

with $k_{0}=\omega / c$ is the vacuum wave vector. The corresponding $\operatorname{Kerr} \theta_{K}^{\prime}$ and Faraday angles $\theta_{F}^{\prime}$ can thus be obtained,

$$
\tan \theta_{K}^{\prime}=\cot \theta_{F}^{\prime}=\frac{2 \sqrt{\frac{\epsilon_{0}}{\mu_{0}}}}{4 \pi\left(\sigma_{x y}^{T}+\sigma_{x y}^{B}\right)} .
$$

At the reflectivity maxima $k L_{z}=\left(N+\frac{1}{2}\right) \pi$, we have

$$
\begin{aligned}
\left\{\begin{array}{c}
r_{S, x x}^{\max } \\
r_{S, x y}^{\max }
\end{array}\right\}= & \frac{1}{\left(\frac{\epsilon}{\mu}\right)^{2}+2 \frac{\epsilon}{\mu}\left(\frac{\epsilon_{0}}{\mu_{0}}-4 \pi \sigma_{x y}^{T} 4 \pi \sigma_{x y}^{B}\right)+\left[\frac{\epsilon_{0}}{\mu_{0}}+\left(4 \pi \sigma_{x y}^{T}\right)^{2}\right]\left[\frac{\epsilon_{0}}{\mu_{0}}+\left(4 \pi \sigma_{x y}^{B}\right)^{2}\right]} \\
& \times\left\{\begin{array}{c}
-\left(\frac{\epsilon}{\mu}\right)^{2}+2 \frac{\epsilon}{\mu} 4 \pi \sigma_{x y}^{T} 4 \pi \sigma_{x y}^{B}+\left[\frac{\epsilon_{0}}{\mu_{0}}-\left(4 \pi \sigma_{x y}^{T}\right)^{2}\right]\left[\frac{\epsilon_{0}}{\mu_{0}}+\left(4 \pi \sigma_{x y}^{B}\right)^{2}\right] \\
-2 \sqrt{\frac{\epsilon_{0}}{\mu_{0}}}\left\{-\frac{\epsilon}{\mu} 4 \pi \sigma_{x y}^{B}+4 \pi \sigma_{x y}^{T}\left[\frac{\epsilon_{0}}{\mu_{0}}+\left(4 \pi \sigma_{x y}^{B}\right)^{2}\right]\right\}
\end{array}\right.
\end{aligned}
$$

and

$$
\left\{\begin{array}{l}
t_{S, x x}^{\max } \\
t_{S, x y}^{\max }
\end{array}\right\}=\frac{e^{-i k_{0} L_{z}}}{\left(\frac{\epsilon}{\mu}\right)^{2}+2 \frac{\epsilon}{\mu}\left(\frac{\epsilon_{0}}{\mu_{0}}-4 \pi \sigma_{x y}^{T} 4 \pi \sigma_{x y}^{B}\right)+\left[\frac{\epsilon_{0}}{\mu_{0}}+\left(4 \pi \sigma_{x y}^{T}\right)^{2}\right]\left[\frac{\epsilon_{0}}{\mu_{0}}+\left(4 \pi \sigma_{x y}^{B}\right)^{2}\right]}\left\{\begin{array}{c}
2 i \sqrt{\frac{\epsilon}{\mu}} \sqrt{\frac{\epsilon_{0}}{\mu_{0}}}\left(\frac{\epsilon}{\mu}+\frac{\epsilon_{0}}{\mu_{0}}-4 \pi \sigma_{x y}^{T} 4 \pi \sigma_{x y}^{B}\right) \\
-2 i \sqrt{\frac{\epsilon}{\mu}} \frac{\epsilon_{0}}{\mu_{0}} 4 \pi\left(\sigma_{x y}^{T}+\sigma_{x y}^{B}\right)
\end{array}\right\} .
$$


The corresponding Kerr $\theta_{K}^{\prime \prime}$ and Faraday angles $\theta_{F}^{\prime \prime}$ can be obtained as

$$
\begin{aligned}
\tan \theta_{K}^{\prime \prime} & =\frac{2 \sqrt{\frac{\epsilon_{0}}{\mu_{0}}}\left\{\frac{\epsilon}{\mu} 4 \pi \sigma_{x y}^{B}-4 \pi \sigma_{x y}^{T}\left[\frac{\epsilon_{0}}{\mu_{0}}+\left(4 \pi \sigma_{x y}^{B}\right)^{2}\right]\right\}}{-\left(\frac{\epsilon}{\mu}\right)^{2}+2 \frac{\epsilon}{\mu} 4 \pi \sigma_{x y}^{T} 4 \pi \sigma_{x y}^{B}+\left[\frac{\epsilon_{0}}{\mu_{0}}-\left(4 \pi \sigma_{x y}^{T}\right)^{2}\right]\left[\frac{\epsilon_{0}}{\mu_{0}}+\left(4 \pi \sigma_{x y}^{B}\right)^{2}\right]}, \\
\tan \theta_{F}^{\prime \prime} & =\frac{\sqrt{\frac{\epsilon_{0}}{\mu_{0}}} 4 \pi\left(\sigma_{x y}^{T}+\sigma_{x y}^{B}\right)}{\frac{\epsilon}{\mu}+\frac{\epsilon_{0}}{\mu_{0}}-4 \pi \sigma_{x y}^{T} 4 \pi \sigma_{x y}^{B}} .
\end{aligned}
$$

From the results Eqs. (E8) and (E9), we can obtain Eqs. (6) and (7) in the main text.

[1] S. M. G. Richard and E. Prange (eds.), The Quantum Hall Effect (Springer-Verlag, New York, 1990).

[2] M. Z. Hasan and C. L. Kane, Colloquium: Topological insulators, Rev. Mod. Phys. 82, 3045 (2010).

[3] X.-L. Qi and S.-C. Zhang, Topological insulators and superconductors, Rev. Mod. Phys. 83, 1057 (2011).

[4] S.-Q. Shen, Topological Insulators, Springer Series of Solid State Science, Vol. 174 (Springer, Heidelberg, 2012).

[5] D. J. Thouless, M. Kohmoto, M. P. Nightingale, and M. den Nijs, Quantized Hall Conductance in a Two-Dimensional Periodic Potential, Phys. Rev. Lett. 49, 405 (1982).

[6] R. B. Laughlin, Quantized Hall conductivity in two dimensions, Phys. Rev. B 23, 5632 (1981).

[7] X.-G. Wen, Theory of the edge states in fractional quantum Hall effects, Int. J. Mod. Phys. 06, 1711 (1992).

[8] Y. Hatsugai, Chern Number and Edge States in the Integer Quantum Hall Effect, Phys. Rev. Lett. 71, 3697 (1993).

[9] C. L. Kane and E. J. Mele, $Z_{2}$ Topological Order and the Quantum Spin Hall Effect, Phys. Rev. Lett. 95, 146802 (2005).

[10] L. Fu and C. L. Kane, Topological insulators with inversion symmetry, Phys. Rev. B 76, 045302 (2007).

[11] X. Chen, A. Tiwari, and S. Ryu, Bulk-boundary correspondence in $(3+1)$-dimensional topological phases, Phys. Rev. B 94, 045113 (2016).

[12] W. A. Benalcazar, B. A. Bernevig, and T. L. Hughes, Quantized electric multipole insulators, Science 357, 61 (2017).

[13] M. Ezawa, Higher-Order Topological Insulators and Semimetals on the Breathing Kagome and Pyrochlore Lattices, Phys. Rev. Lett. 120, 026801 (2018).

[14] D. Călugăru, V. Juričić, and B. Roy, Higher-order topological phases: A general principle of construction, Phys. Rev. B 99, 041301(R) (2019).

[15] F. Schindler, A. M. Cook, M. G. Vergniory, Z. Wang, S. S. Parkin, B. A. Bernevig, and T. Neupert, Higher-order topological insulators, Sci. Adv. 4, eaat0346 (2018).

[16] F. Schindler, Z. Wang, M. G. Vergniory, A. M. Cook, A. Murani, S. Sengupta, A. Y. Kasumov, R. Deblock, S. Jeon, I. Drozdov et al., Higher-order topology in bismuth, Nat. Phys. 14, 918 (2018).

[17] W. A. Benalcazar, B. A. Bernevig, and T. L. Hughes, Electric multipole moments, topological multipole moment pumping, and chiral hinge states in crystalline insulators, Phys. Rev. B 96, 245115 (2017).

[18] Z. Song, Z. Fang, and C. Fang, $(d-2)$-Dimensional Edge States of Rotation Symmetry Protected Topological States, Phys. Rev. Lett. 119, 246402 (2017).
[19] J. Langbehn, Y. Peng, L. Trifunovic, F. von Oppen, and P. W. Brouwer, Reflection-Symmetric Second-Order Topological Insulators and Superconductors, Phys. Rev. Lett. 119, 246401 (2017).

[20] E. Khalaf, Higher-order topological insulators and superconductors protected by inversion symmetry, Phys. Rev. B 97, 205136 (2018).

[21] M. Geier, L. Trifunovic, M. Hoskam, and P. W. Brouwer, Second-order topological insulators and superconductors with an order-two crystalline symmetry, Phys. Rev. B 97, 205135 (2018).

[22] C. Fang and L. Fu, New classes of topological crystalline insulators having surface rotation anomaly, Sci. Adv. 5, eaat 2374 (2019).

[23] L. Trifunovic and P. W. Brouwer, Higher-Order Bulk-Boundary Correspondence for Topological Crystalline Phases, Phys. Rev. X 9, 011012 (2019).

[24] C. Yue, Y. Xu, Z. Song, H. Weng, Y.-M. Lu, C. Fang, and X. Dai, Symmetry-enforced chiral hinge states and surface quantum anomalous Hall effect in the magnetic axion insulator $\mathrm{Bi}_{2-x} \mathrm{Sm}_{x} \mathrm{Se}_{3}$, Nat. Phys 15, 577 (2019).

[25] S. H. Kooi, G. van Miert, and C. Ortix, Inversion-symmetry protected chiral hinge states in stacks of doped quantum Hall layers, Phys. Rev. B 98, 245102 (2018).

[26] G. van Miert and C. Ortix, Higher-order topological insulators protected by inversion and rotoinversion symmetries, Phys. Rev. B 98, 081110(R) (2018).

[27] K. Plekhanov, F. Ronetti, D. Loss, and J. Klinovaja, Hinge states in a system of coupled Rashba layers, Phys. Rev. Research 2, 013083 (2020).

[28] E. Khalaf, H. C. Po, A. Vishwanath, and H. Watanabe, Symmetry Indicators and Anomalous Surface States of Topological Crystalline Insulators, Phys. Rev. X 8, 031070 (2018).

[29] J. Kruthoff, J. de Boer, J. van Wezel, C. L. Kane, and R.-J. Slager, Topological Classification of Crystalline Insulators through Band Structure Combinatorics, Phys. Rev. X 7, 041069 (2017).

[30] H. C. Po, A. Vishwanath, and H. Watanabe, Symmetry-based indicators of band topology in the 230 space groups, Nat. Commun. 8, 50 (2017).

[31] S. Ono and H. Watanabe, Unified understanding of symmetry indicators for all internal symmetry classes, Phys. Rev. B 98, 115150 (2018).

[32] H. Watanabe, H. C. Po, and A. Vishwanath, Structure and topology of band structures in the 1651 magnetic space groups, Sci. Adv. 4, eaat8685 (2018). 
[33] F. Tang, H. C. Po, A. Vishwanath, and X. Wan, Comprehensive search for topological materials using symmetry indicators, Nature (London) 566, 486 (2019).

[34] F. Tang, H. C. Po, A. Vishwanath, and X. Wan, Efficient topological materials discovery using symmetry indicators, Nat. Phys. 15, 470 (2019).

[35] Y. Tanaka, R. Takahashi, and S. Murakami, Appearance of hinge states in second-order topological insulators via the cutting procedure, Phys. Rev. B 101, 115120 (2020).

[36] Y. Tanaka, R. Takahashi, T. Zhang, and S. Murakami, Theory of inversion $\mathbb{Z}_{4}$ protected topological chiral hinge states and its applications to layered antiferromagnets, Phys. Rev. Research 2, 043274 (2020).

[37] R. Takahashi, Y. Tanaka, and S. Murakami, Bulk-edge and bulkhinge correspondence in inversion-symmetric insulators, Phys. Rev. Research 2, 013300 (2020).

[38] W.-Y. Shan, H.-Z. Lu, and S.-Q. Shen, Effective continuous model for surface states and thin films of three-dimensional topological insulators, New J. Phys. 12, 043048 (2010).

[39] M. S. Dresselhaus, G. Dresselhaus, and A. Jorio, Group Theory: Application to the Physics of Condensed Matter (SpringerVerlag, Berlin, 2008).

[40] B. Kang, K. Shiozaki, and G. Y. Cho, Many-body order parameters for multipoles in solids, Phys. Rev. B 100, 245134 (2019).

[41] W. A. Wheeler, L. K. Wagner, and T. L. Hughes, Many-body electric multipole operators in extended systems, Phys. Rev. B 100, 245135 (2019)

[42] C.-A. Li, B. Fu, Z.-A. Hu, J. Li, and S.-Q. Shen, Topological Phase Transitions in Disordered Electric Quadrupole Insulators, Phys. Rev. Lett. 125, 166801 (2020).

[43] S. Ono, L. Trifunovic, and H. Watanabe, Difficulties in operator-based formulation of the bulk quadrupole moment, Phys. Rev. B 100, 245133 (2019).

[44] R.-L. Chu, J. Shi, and S.-Q. Shen, Surface edge state and half-quantized Hall conductance in topological insulators, Phys. Rev. B 84, 085312 (2011).

[45] X.-L. Qi, T. L. Hughes, and S.-C. Zhang, Chiral topological superconductor from the quantum Hall state, Phys. Rev. B 82, 184516 (2010).

[46] F. Wilczek, Two Applications of Axion Electrodynamics, Phys. Rev. Lett. 58, 1799 (1987).
[47] T. L. Hughes, E. Prodan, and B. A. Bernevig, Inversionsymmetric topological insulators, Phys. Rev. B 83, 245132 (2011).

[48] A. M. Turner, Y. Zhang, R. S. Mong, and A. Vishwanath, Quantized response and topology of magnetic insulators with inversion symmetry, Phys. Rev. B 85, 165120 (2012).

[49] J. Maciejko, X.-L. Qi, H. D. Drew, and S.-C. Zhang, Topological Quantization in Units of the Fine Structure Constant, Phys. Rev. Lett. 105, 166803 (2010).

[50] W.-K. Tse and A. H. MacDonald, Magneto-optical Faraday and Kerr effects in topological insulator films and in other layered quantized Hall systems, Phys. Rev. B 84, 205327 (2011).

[51] W.-K. Tse and A. MacDonald, Giant Magneto-Optical Kerr Effect and Universal Faraday Effect in Thin-Film Topological Insulators, Phys. Rev. Lett. 105, 057401 (2010).

[52] K. N. Okada, Y. Takahashi, M. Mogi, R. Yoshimi, A. Tsukazaki, K. S. Takahashi, N. Ogawa, M. Kawasaki, and Y. Tokura, Terahertz spectroscopy on Faraday and Kerr rotations in a quantum anomalous Hall state, Nat. Commun. 7, 12245 (2016).

[53] V. Dziom, A. Shuvaev, A. Pimenov, G. Astakhov, C. Ames, K. Bendias, J. Böttcher, G. Tkachov, E. Hankiewicz, C. Brüne et al., Observation of the universal magnetoelectric effect in a 3D topological insulator, Nat. Commun. 8, 15197 (2017).

[54] M. Sitte, A. Rosch, E. Altman, and L. Fritz, Topological Insulators in Magnetic Fields: Quantum Hall Effect and Edge Channels with a Nonquantized $\theta$ Term, Phys. Rev. Lett. 108, 126807 (2012).

[55] J. Wang, B. Lian, X.-L. Qi, and S.-C. Zhang, Quantized topological magnetoelectric effect of the zero-plateau quantum anomalous Hall state, Phys. Rev. B 92, 081107(R) (2015).

[56] X.-L. Qi, T. L. Hughes, and S.-C. Zhang, Topological field theory of time-reversal invariant insulators, Phys. Rev. B 78, 195424 (2008).

[57] A. M. Essin, J. E. Moore, and D. Vanderbilt, Magnetoelectric Polarizability and Axion Electrodynamics in Crystalline Insulators, Phys. Rev. Lett. 102, 146805 (2009).

[58] C. Fang, M. J. Gilbert, and B. A. Bernevig, Bulk topological invariants in noninteracting point group symmetric insulators, Phys. Rev. B 86, 115112 (2012). 